\title{
Herramientas para la toma de decisiones en Sistemas Alimentarios
}

Helena Posthumus, Jonne Bosselaar y Herman Brouwer

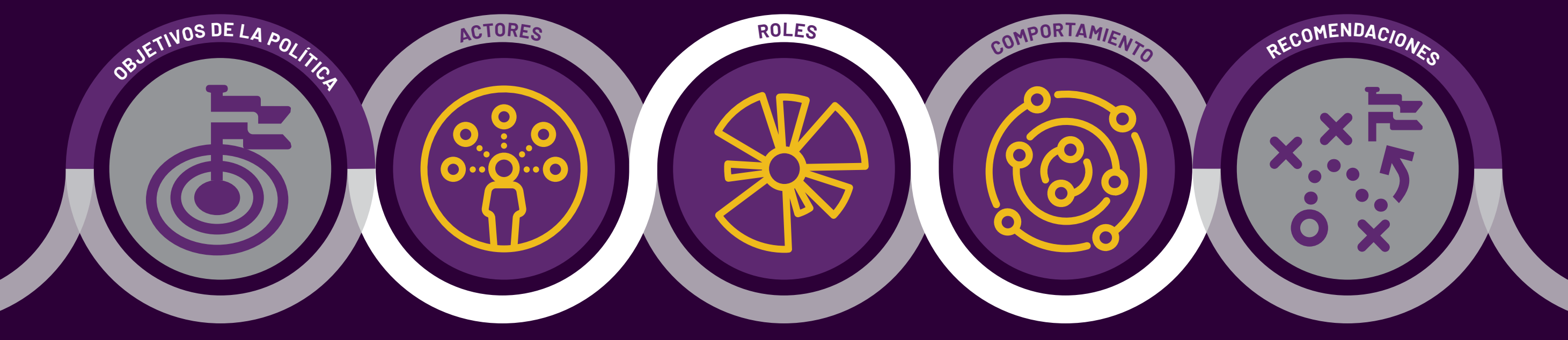




\section{Contenidos}

\section{¿Qué es Food System Decision} Support Toolbox?

- Presentación general

- Proceso de análisis del sistema alimentario

- Presentación para legisladores

- Definición de un objetivo del sistema alimentario

\section{Análisis del sistema} alimentario

Presentación para analistas del sistema alimentario

Un análisis con tres principios

Principio de calidad A: Pensamiento del sistema

- Principio de calidad B: Implicación de los grupos de interés

ه Principio de calidad C: Equidad e inclusión

- La Herramienta

1. Actores del sistema

2. Características del sistema

3. Comportamiento del sistema

\section{Del análisis}

a la acción

- El análisis del sistema alimentario, en acción

Panorámica general de las fuentes

- Cierre 


\section{¿Qué es la herramienta food system decision support?}

Índice

- Presentación general

- Proceso de análisis del sistema alimentario

- Presentación para legisladores

- Definición de un objetivo del sistema alimentario 


\section{Presentación general}

\section{¿Por qué una herramienta como esta?}

Esta herramienta se ha creado con el objetivo de comparar los distintos métodos y herramientas que podemos utilizar para analizar el sistema alimentario. Se basa específicamente en sistemas pensados para el análisis del sistema alimentario con el fin de dar recomendaciones prácticas que hagan posible un cambio sistémico. Describe tanto el proceso de un análisis del sistema alimentario como un conjunto de herramientas que se pueden utilizar en distintas etapas.

La herramienta no ofrece una receta paso a paso para analizar el sistema alimentario, es más bien un libro de cocina que trata de inspirar a todo aquel a quien le interese hacerlo, independientemente de si se trata de un legislador, un profesional o un investigador. Ayuda a tomar decisiones estratégicas informadas sobre el diseño de políticas o intervenciones en los ámbitos de la alimentación, la agricultura o el desarrollo. El análisis del sistema alimentario que se describe en esta guía se entiende, por tanto, como una evaluación ex-ante con el fin de fundamentar las políticas, estrategias o programas que traten de contribuir a un cambio estructural en el sistema alimentario.

\section{¿A quién va dirigida la herramienta?}

Trata de servir como fuente de inspiración a diversos usuarios:

- Legisladores que quieran realizar un análisis del sistema alimentario para fundamentar las nuevas políticas y los procesos de previsión.

Investigadores que quieran utilizar una combinación de métodos de análisis y participación para entender mejor las relaciones causa-efecto del sistema alimentario. ONG que quieran llevar a cabo intervenciones holísticas para mejorar los resultados del sistema alimentario (como la seguridad alimentaria y de la nutrición. ocupándose de cuellos de botella concretos.
Desde el principio, necesitará tener claro quién lidera el análisis y quién decidirá (o se hará cargo) de las recomendaciones finales. Somos firmes defensores de incluir a los grupos de interés locales en el sistema alimentario. Los invitados que elijamos para que se

sienten con nosotros en la misma mesa a lo largo de todo el proceso van a influir en las conclusiones del análisis, las recomendaciones de intervención y el nivel de presencia o aceptación de los grupos de interés locales. Por lo tanto, recomendamos que participe un amplio número de grupos de interés, de modo que se incorporen al análisis sus conocimientos tácitos y con el fin de lograr un entendimiento conjunto del sistema alimentario y la responsabilidad en cuanto a las recomendaciones (véase el apartado Implicación de los grupos de interés). Las voces a las que menos se suele escuchar pueden aportar valiosos conocimientos.

\section{¿Cómo se usa la herramienta?}

Esta herramienta describe el proceso de análisis del sistema alimentario, los principios de calidad que creemos que debería guiar ese análisis, las distintas piezas del análisis y las herramientas que podemos utilizar para llevarlo a cabo. En el marco del proceso de análisis, distinguimos tres piezas: actores del sistema características del sistema y comportamiento del sistema. Cada una de ellas se centra en una dimensión distinta del sistema, pero no hace falta seguir este orden en el análisis. En cambio, sí se pueden analizar estas tres dimensiones en paralelo o de manera reiterada, para profundizar más en problemas o partes concretos del sistema alimentario. Por ejemplo, la primera fase podría consistir en un análisis somero de los principales problemas y su causa raíz, seguido de una segunda donde se analizarian las posibles palancas con más detalle.

a herramienta no es exhaustiva: hay muchas otras que pueden emplearse en el análisis. Hemos clasificado un selecto grupo de herramientas en tres categorías: herramientas de concienciacion, herramientas de exploración y herramientas de análisis (para conocer más detalles, véase Presentación para analistas del sistema alimentario). De este modo, el usuario puede optar entre un análisis superficial, un proyecto de investigación interdisciplinario o cualquier opción intermedia. Para llevar a cabo un análisis superficial, por ejemplo, puede organizar un taller con diversos grupos de interés y elegir varias herramientas de concienciación para alentar el debate y recopilar perspectivas sobre el sistema alimentario. Del mismo modo, pueden utilizarse herramientas de exploración para profundizar en aquellas áreas en las que haya descubierto lagunas de conocimiento o puntos de vista que entran en conflicto entre los distintos grupos de interés. Si desea obtener un conocimiento más en profundidad, puede combinar herramientas de exploración y análisis para realizar un estudio integral del sistema alimentario.

Las tres piezas del análisis y los principios de calidad que se describen en esta herramienta son esenciales para analizar el sistema alimentario. Usted decide qué herramientas utilizar, en función del contexto y la disponibilidad de sus recursos. 


\section{Análisis del sistema alimentario: el proceso}

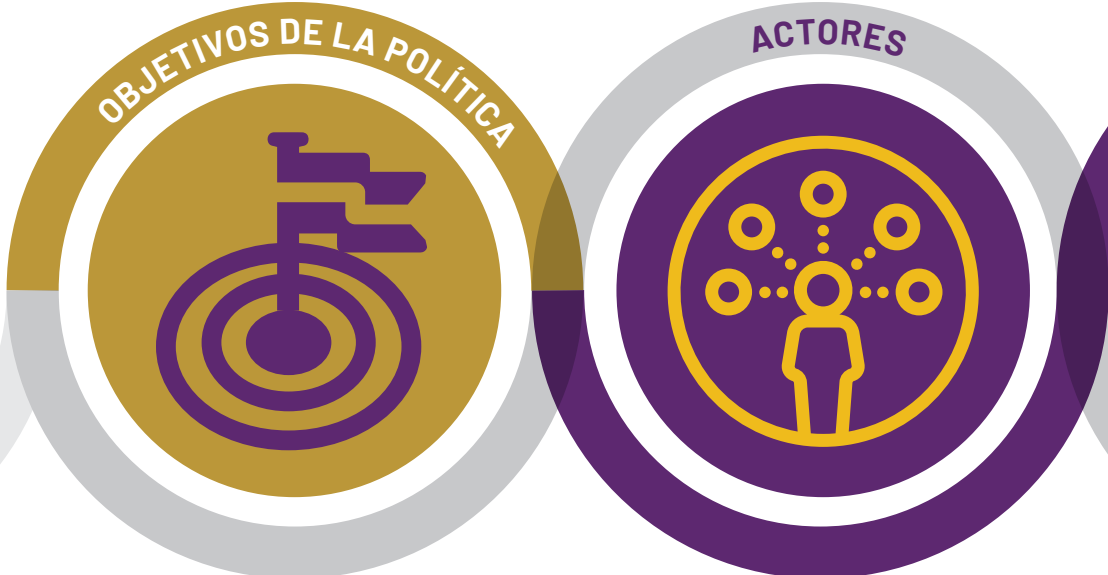

Objetivos de la política

El punto de partida de cualquier análisis es el objetivo o la cuestión política más amplia que se deba tratar, incluidos el nivel de intervención o el grupo objetivo. Se trata de responder a la pregunta: ¿por qué hacemos este análisis del sistema alimentario? Ese objetivo determina la dirección y los límites del análisis del sistema alimentario.
Actores del sistema alimentario

Los actores del sistema incluyen a todas las personas, grupos y organizaciones que, de una forma u otra, están presentes en el sistema alimentario o les afecta. A través de sus acciones e interacciones, construyen, reforman o bloquean el sistema alimentario. Identificar los

intereses y la influencia de los distintos actores, así como las dinámicas de poder subyacentes, ayuda a fundamentar sus estrategias para fomentar la participación.
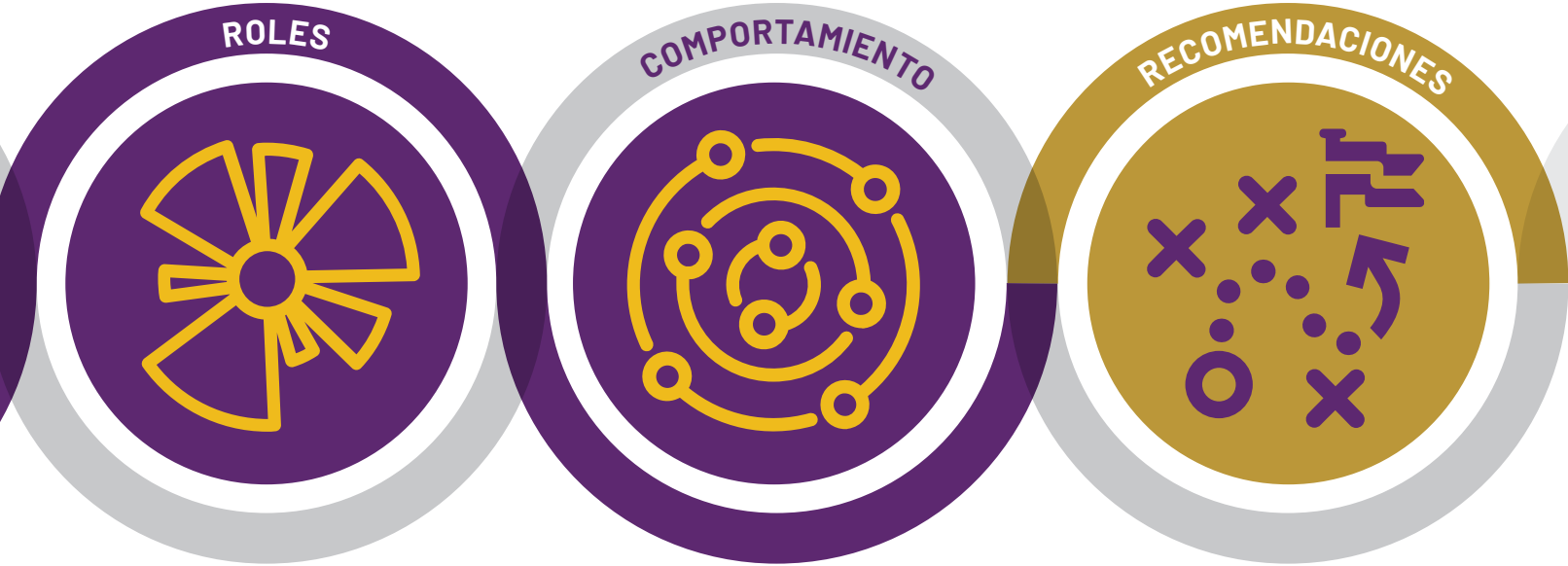

Características del sistema alimentario

Un sistema alimentario tiene muchos elementos distintos, entre ellos, motores, actividades y resultados. Para entenderlo, es necesario conocer las tendencias de estas piezas sobre la base de indicadores clave, así como las sinergias y contrapesos entre ellas.
Comportamiento del sistema alimentario

Los distintos elementos que componen el sistema alimentario no funcionan de manera independiente. Conforme interactúan, los sistemas revelan patrones de comportamiento que determinan los resultados. El examen de las relaciones causales hace más fácil entender cómo se comporta e sistema e identificar palancas de cambio.
Recomendaciones

Esta última etapa explora el margen que existe para realizar posibles intervenciones dirigidas a esas palancas. Las conclusiones del análisis del sistema alimentario

se combinan para realizar recomendaciones prácticas. 


\section{Casos de usuarios}

La herramienta se puede utilizar de distintas formas, dependiendo del objetivo y el contexto del análisis del sistema alimentario. Estos son los casos de dos usuarios, que sirven de ejemplos sobre cómo utilizarla.

Ejemplo A: Una embajada de los Países Bajos quiere poner en marcha un nuevo programa para evitar la pérdida de alimentos en las cadenas de suministro de alimentación desde las zonas rurales a las urbanas, con el fin de mejorar la seguridad alimentaria y de la nutrición, asi como la generación de ingresos en el país $X$. Los casos de alimentos echados a perder (tras la cosecha) han suscitado interés en este asunto y la embajada trata de averiguar qué intervenciones podrían tener un impacto potencial significativo. Aunque su personal dispone de conocimientos generales sobre el sector agrario de este país, desconocen cómo funcionan las cadenas de suministro de alimentos desde las zonas rurales a las urbanas, y cómo influyen sobre ellas las dinámicas del sistema alimentario en general. Por lo tanto, la embajada quiere llevar a cabo un análisis del sistema alimentario para conocer posibles ámbitos de intervención que podrían lograr diversos objetivos (reducción de la pérdida de alimentos, empleo juvenil, seguridad de la nutrición, generación de ingresos).

Ejemplo B: Una ONG internacional ha estado trabajando en el campo de la seguridad alimentaria y de la nutrición y el desarrollo de la cadena de valor durante muchos años. Ahora, tienen previsto ampliar sus actividades al país $Y$, que es un Estado frágil. Es la primera vez que la ONG va a trabajar en un Estado frágil, y se dan cuenta de que tendrán que recurrir a nuevos enfoques y colaboradores para que su intervención resulte exitosa. Para entender mejor las causas subyacentes de la inseguridad alimentaria, las dinámicas de poder y los factores que explican el conflicto, así como posibles grupos de interés con los que colaborar, la ONG decide hacer primero un análisis del sistema alimentario.

\section{Ejemplo A}

Solicitante: embajada de los Países Bajos Objetivo: pérdida de alimentos en las

cadenas de suministro de alimentos desde zonas rurales hacia zonas urbanas

Limites: corredores comerciales entre zonas rurales $\mathrm{y}$ urbanas $\mathrm{y}$ actores del pais $\mathrm{X}$
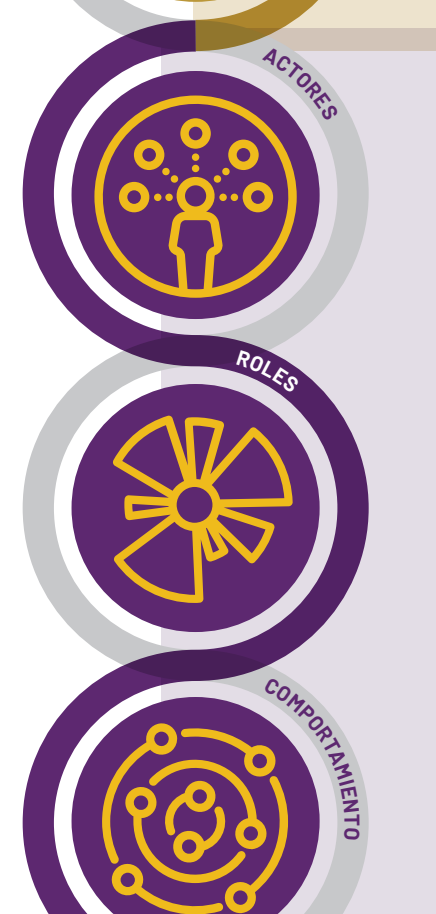
$\downarrow$

\section{Mapeo de los grupos de interés}

Evaluación rápida para identificar grupos de

interés clave

- Caracteristicasy roles

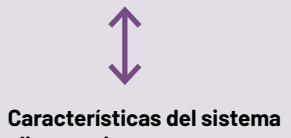

alimentario

Delimitación del estudio

- Coste mínimo de una dieta

saludable

- Herramienta de la caden

de valor que tiene en cuen-

ta la nutrición e incorpor

la perdida

análisis

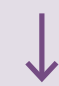

Comportamiento del sistem r con los grupos de interes

- Gráfico 00

del tiempo

(n)

innovación

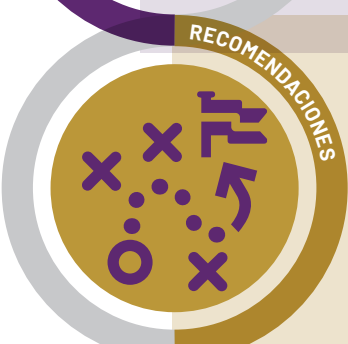

y roles de los grupos de interés

ento a lo largo

Ejemplo B

Solicitante: ONG internacional

Objetivo: intervención SAN en un Estado frágil

Limites: escala nacional en el pais $Y$

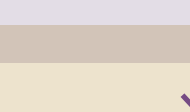

Definición de las condiciones de referencia con los accionistas

- Priorización y clasificación de ideas conomia politica debate grupal

clave Las cinco A

Mapeo de los grupos de interés Evaluación rápida para ide

- Identificación de los grupos de

interés

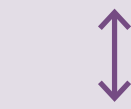

$\downarrow$

Caracteristicas del sistema aliment

- Repaso de la litera个1 $\quad$ Entrevistas

sistema

Comportamiento de sistema grupos
clave

- Arquetipos de - Arquetipos d

- Ruptura del ciclo - Diagramas de
circuito causal
- Arquetipos de - Arquetipos tiquetas
Comportamiento del Comportamie

\section{Actores del sistema} Estudio de gabinete clave

- Mapeo de poder - Poder y comporta-

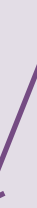

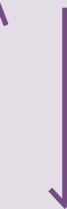

del sistema

Estudio de gabinete con informantes

clave

- Planificación para el éxito - Análisis swot grupos de interés aller con los grupos de interés - Matriz de intereses e influencia - Clasificación de poder

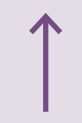

Caracteristicas del sistimitación del estudo - Análisis de las brechas nutricionales - Evaluaciones rápidas del sector - Repaso de la literatura publicada - Análisis de políticas $\pi$ Comportamiento del sistem Taller con
interés - Ruptura del ciclo - Rueda de futuros

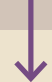

Selección de las áreas de intervención (área geogra Diseño de programa con los grupos de interes clave

Capuestas Cambio -Planificación de acciones 


\section{Presentación para legisladores}

Independientemente de si trabaja en un ministerio, un organismo del Gobierno, una agencia de donantes, una firma de inversión o una ONG, si usted está activo en los ámbitos de la alimentación, la agricultura y el desarrollo, es probable que su trabajo incluya tratar de responder a los desafíos que plantean los sistemas alimentarios que provocan resultados no deseados, como la inseguridad alimentaria, la desigualdad, el deterioro del medioambiente o el desempleo. Entender los diversos desafíos y oportunidades que presenta el sistema alimentario es fundamental para poder poner en marcha intervenciones eficaces. Esta herramienta le ofrece los mimbres que necesita para llevar a cabo un análisis del sistema alimentario que puede utilizarse para hacer recomendaciones sobre políticas y programas.

El enfoque del Sistema Alimentario se utiliza cada vez más como marco para entender y dar forma a intervenciones y políticas estratégicas relativas a la agricultura y la seguridad alimentaria y de la nutrición. Según lo define el Reto del Hambre Cero del Secretario General de la ONU, un sistema alimentario es un sistema que "está formado por todos los elementos (medio ambiente, población recursos, procesos, instituciones, infraestructuras, etc.) y actividades relacionadas con la producción, procesamiento, distribución, preparación y consumo de alimentos, así como los resultados de estas actividades en el crecimiento socioeconómico, y la sostenibilidad ambiental". Sin embargo, transformar las perspectivas y la inspiración en cuanto al sistema alimentario en intervenciones eficaces sigue suponiendo un desafío. El objetivo de esta herramienta es crear un método de análisis del sistema alimentario que dé como resultado recomendaciones prácticas que puedan ayudar a que se produzcan cambios sistémicos. No se espera que los legisladores hagan ellos mismos el análisis, pero sí pueden pedírselo a un tercero.
Un buen análisis del sistema alimentario debería incluir, al menos, los siguientes elementos:

- Conocimiento de las tendencias de los indicadores clave que representan los resultados, motores y actividades deseados, como la seguridad alimentaria, las actividades económicas, los efectos medioambientales o el bienestar.

- Perspectivas sobre los procesos del sistema que revelen los efectos de interacción con el sistema alimentario que den resultados deseados o indeseados.

- Conocimiento de la influencia y los intereses de actores concretos que condicionan el sistema alimentario, o a quienes este les afecta. Esto incluye perspectivas sobre las dinámicas de poder o las distintas formas en las que los diferentes actores pueden interactuar (o no) y se ven afectados (tanto positiva como negativamente).

- Estas perspectivas permiten identificar palancas de cambio del sistema que pueden fundamentar las recomendaciones estratégicas.

Las perspectivas anteriores deberían ser el resultado de un proceso facilitado de implicación de los grupos de interés, en el que se tengan en cuenta las perspectivas de diversos grupos de interés, que se contextualicen en las tendencias más generales. Eso exige facilitadores y analistas experimentados que sean capaces de analizar las tendencias y procesos a distintos niveles e implicarse con diversos tipos de grupos de interés.

\section{Encargar un buen análisis del sistema alimentario}

Cualquier legislador que esté pensando en encargar un buen análisis del sistema alimentario debería esperar lo siguiente:

- El análisis ofrece perspectivas actualizadas sobre la situación y las tendencias actuales del sistema alimentario que se analizan, incluidas las áreas de interés.

- El análisis adopta una perspectiva sistemática, por lo que también analiza cómo influyen entre sí los distintos motores, factores, procesos y actores.

- El análisis incluye los motivos y la influencia de los distintos grupos de actores, y quién se queda dentro y fuera de las prestaciones.

- Diversos grupos de interés están activamente comprometidos con el análisis.

- El análisis cuenta con una perspectiva aplicada que genera recomendaciones prácticas sobre cómo es mejor intervenir en el sistema alimentario, que incluyen con quién colaborar para lograr los objetivos de la política.

El punto de partida de cualquier análisis es la meta de la política o el objetivo sobre el sistema alimentario que se deba tratar, incluidos el nivel de intervención o el grupo objetivo. Esta meta política más amplia definirá los límites del análisis del sistema alimentario. Unos límites del sistema alimentario bien definidos permitirán un análisis más en profundidad; si no se definen correctamente, el análisis será somero. 


\section{Definición de un objetivo del sistema alimentario}

Para obtener recomendaciones prácticas debe ser explícito en cuanto a qué tipo de objetivos genéricos prevé conseguir y para quién. Realizar un análisis del sistema alimentario sin un fin último en mente puede llevar a que su alcance sea insignificante y adolezca de vaguedad. Aunque el objetivo debe indicar el elemento central de análisis del sistema alimentario, debe ser lo bastante amplio como para estar abierto a distintas estrategias, políticas o intervenciones con el fin de lograrlo.

\section{Resultados del sistema alimentario}

Distinguimos tres formas de resultados del sistema alimentario en el análisis del sistema alimentario: resultados socioeconómicos, resultados de seguridad alimentaria y de la nutrición (SAN) y resultados medioambientales.

\section{Resultados de seguridad alimentaria y de la nutrición:} La seguridad alimentaria y de la nutrición (SAN) suele ser el punto de partida de un análisis del sistema alimentario. Entender las dinámicas que rodean a la SAN en los distintos grupos sociales guiará el análisis posterior. La SAN es el resultado de la disponibilidad, el acceso (que incluye la asequibilidad), el uso y la estabilidad de los alimentos. Esto incluye, por ejemplo, los patrones de consumo, el valor nutricional de las dietas, la infraestructura del mercado, la producción, almacenamiento y el comercio de alimentos, así como las fluctuaciones estacionales en su disponibilidad. Un objetivo relacionado con los resultados de la SAN podría ser: mejorar las dietas saludables de [grupo objetivo].

Resultados socioeconómicos: El sistema alimentario genera resultados socioeconómicos, tales como salud, empleo y riqueza, pero también ingresos y condiciones de vida para grupos objetivo concretos. Resulta evidente que el sector agroalimentario es uno de los principales motores económicos y de bienestar social de un país. Una gran cantidad de hogares de todo el mundo viven

del sector agroalimentario, desde quienes practican la agricultura de subsistencia hasta quienes trabajan en la producción de alimentos a escala industrial. Asimismo, el comportamiento del sistema alimentario influye sobre los que salen ganando y los que se quedan fuera. Eso afecta a los niveles de pobreza e igualdad entre ciudadanos. Puesto que nuestro objetivo es transformar los sistemas alimentarios de modo que funcionen para la mayoría, resulta fundamental conocer sus resultados socioeconómicos. Un objetivo relacionado con los resultados socioeconómicos podría ser: incrementar los ingresos de los hogares de [ grupo objetivo] en [zona geográfica]

Resultados medioambientales: Las actividades de los sistemas alimentarios suelen competir por aquellos recursos naturales comunes que se ven amenazados por las actividades humanas. El papel de la agricultura y el consumo de alimentos en el deterioro de la salud de nuestro planeta está sobradamente acreditado. La transición hacia un sistema alimentario sostenible no solo consiste en producir alimentos nutritivos y respaldar las condiciones de vida de quienes viven de él, también trata de lidiar con el deterioro medioambiental y el cambio climático. El análisis del sistema alimentario debería buscar sendas hacia unos resultados más regeneradores y sostenibles. Un objetivo relacionado con los resultados medioambientales podría ser: prevención del deterioro del suelo y la contaminación del agua en [zona geográfica].

Herramientas para definir un objetivo del sistema alimentario

\begin{tabular}{l|l} 
Cuadro enriquecido & Evaluación de necesidades
\end{tabular}

Visualice el futuro que se desea

y los desafíos que plantea su

materialización, incluidos los actores

y factores pertinentes que afecten a esta cuestión.

\section{Limites del sistema}

Un sistema alimentario no se ciñe casi nunca a una zona geográfica concreta. Del mismo modo, un sistema alimentario también está integrado en, y se ve afectado por, otros sistemas humanos o naturales, por lo que, en realidad, no es posible trazar una frontera clara donde empieza y acaba el sistema alimentario. No obstante, es importante elegir los límites del sistema (por ejemplo, en función de la geografía, los resultados o los grupos objetivo), de modo que no se pierda el centro de atención del análisis. A la hora de delimitar sistema, el objetivo del análisis del sistema alimentario debería ser lo fundamental, pero también se deben tener en cuenta los recursos disponibles (tiempo, financiación). En el transcurso del análisis, es posible que quiera volver a los límites y reajustarlos si es necesario.

\section{Grupos objetivo y diversidad}

Los resultados del sistema alimentario nunca son iguales para los distintos grupos que conforman una sociedad. Por lo tanto, el objetivo puede hacer referencia a un grupo objetivo concreto. Si es el caso, debería ser uno de los puntos en el que centrar el análisis del sistema alimentario. Aunque no haya un grupo objetivo específico no deja de ser importante tener en cuenta la diversidad de la sociedad, ya que las políticas nunca son neutras $y$ afectan de manera inherentemente distinta a los diferentes grupos sociales.
Evaluar las necesidades del grupo objetivo para formular el objetivo estratégico.
Definición de problemas Aclarar el problema sobre el que se trabaja haciendo cinco preguntas. Ayudará al grupo a centrar las ideas en una misma dirección. 


\section{Analizar el sistema alimentario}

Índice

- Presentación para analistas del sistema alimentario

- Un análisis con tres principios

- Principio de calidad A: Pensamiento del sistema

- Principio de calidad B: Implicación de los grupos de interés

- Principio de calidad C: Equidad e inclusión

La Herramienta

1. Actores del sistema

2. Características del sistema

3. Comportamiento del sistema 


\section{Presentación para analistas del sistema alimentario}

\section{Una herramienta para analizar y ayudar a adoptar estrategias de cara al sistema alimentario}

El objetivo de esta herramienta es realizar un análisis integral del sistema alimentario que sea útil para quien necesite tomar decisiones informadas y definir su estrategia en el contexto de un sistema alimentario complejo. Esta estrategia puede servir para lidiar con diversos resultados de los sistemas alimentarios, los resultados de seguridad alimentaria y de la nutrición y los resultados medioambientales. El análisis muestra la situación actual del sistema alimentario y las tendencias (características del sistema) la interrelación entre las distintas partes del mismo y sus palancas (comportamiento del sistema) y cómo los actores, las relaciones de poder y las estructuras de gobernanza influyen en el sistema alimentario (actores del sistema). El análisis puede ayudarle a definir una estrategia, política o proceso de previsión para hacer cambios en un sistema alimentario con el fin de lograr los resultados deseados.

\section{Cómo usar la herramienta}

Esta herramienta ofrece herramientas de tres tipos:

(- Concienciación: para aumentar el conocimiento de algunas de las cuestiones en juego. Muchas de estas herramientas pueden utilizarse en talleres con diversos grupos de interés que cuenten con facilitadores. Estas herramientas exigen experiencia en la facilitación de talleres y el conocimiento previo del sistema alimentario pero su coste es bajo.

OO Exploración: estas herramientas profundizan un poco más en cuestiones concretas y exigen algunos recursos de dedicación para la recopilación y el análisis de datos. Estas herramientas son una mezcla de estudio de gabinete y trabajo de campo que exigen experiencia en la investigación y el análisis (cualitativo). El trabajo de campo y el análisis exigirán que se dediquen a ellos ciertos recursos económicos. inl Análisis: estas herramientas son en su mayoría métodos de análisis que se pueden utilizar para profundizar y realizar análisis detallados de cuestiones concretas en el marco de un proyecto de investigación financiado sobre los sistemas alimentarios. Las herramientas son una mezcla de métodos de análisis y técnicas de modelado que exigen competencias concretas en investigación y una inversión de tiempo y dinero considerable.

Las herramientas que incluye esta herramienta se han probado y testado en diferentes entornos. Por lo tanto, nos remitidos a las correspondientes fuentes para ofrecer una descripción más detallada de cada una de ellas.

Con cada una de las herramientas indicamos el tipo de modalidad:

88. Taller con grupos de interés o informantes
Entrevistas con informantes clave

() Análisis realizado por los investigadores

Esto ayuda también a indicar rápidamente qué herramientas pueden combinarse fácilmente, por ejemplo, en un taller o una sesión. La participación de los grupos de interés es clave en todas las herramientas para asegurar que el conocimiento que se genere se crea de forma conjunta y se orienta a la práctica.

El análisis del sistema alimentario exige conocimientos expertos interdisciplinares sobre el sistema

Como el propio término sistema alimentario implica el análisis se centra en el conjunto del sistema y las relaciones entre los distintos motores y resultados del mismo. Un análisis del sistema alimentario exige un enfoque interdisciplinar, que exige a su vez un conocimiento experto en el pensamiento del sistema, los enfoques participativos y disciplinas académicas concretas. Son necesarias una serie de competencias, desde la facilitación de talleres con diversos grupos de interés hasta la realización de análisis económicos, sociales o biofísicos. La mejor forma de hacer un análisis integral es a través de un equipo interdisciplinar de analistas equipados con experiencias, procedencias y competencias distintas pero relevantes. Asegúrese de contar con un grupo diverso de expertos internacionales y locales en el equipo.

Puede ajustar el análisis del sistema alimentario que haga en función de su tiempo y presupuesto

Hacer análisis del sistema alimentario puede consumir gran cantidad de tiempo. No obstante, hay distintos niveles de análisis posibles, dependiendo del objetivo, las brechas de conocimiento y los recursos disponibles. Ofrecemos una estimación del tiempo necesario para ejecutar cada una de estas herramientas. Esta indicación temporal hace referencia al tiempo de actividad que hace falta para utilizar la herramienta, incluido el necesario para la preparación o la presentación de informes. El rango

temporal es bastante amplio para algunas herramientas. En función del objetivo del estudio y el tiempo y los recursos económicos de los que se disponga, el analista podrá elegir la profundidad con la que quiera utilizar la herramienta. Si ya existen conocimientos suficientes sobre determinados elementos del sistema alimentario, algunos puntos podrán saltarse o tratarse marginalmente. Habrá que tener en cuenta determinados equilibrios por ejemplo, entre el rigor académico, la calidad de la implicación o el trabajo propio de los grupos de interés y la duración del análisis. 


\section{Un análisis con tres principios}

La herramienta se divide en tres piezas de análisis: características del sistema, comportamiento del sistema y actores del sistema. Estas tres piezas juntas ofrecerán la perspectiva necesaria para lograr el objetivo que se haya formulado y fundamentar el diseño de una política o una estrategia de intervención. En función del conocimiento existente sobre el sistema alimentario y los problemas que se vayan a tratar, es posible que haya partes de la herramienta más relevantes que otras. También es posible que sea necesaria una cierta reiteración para tratar brechas de conocimiento concretas.

\section{Actores del sistema}

El funcionamiento de un sistema alimentario depende en buena medida de los actores que están presentes en él. Los actores del sistema incluyen a todas las personas, grupos y organizaciones que, de una forma u otra, están presentes en el sistema alimentario. Con sus acciones e interacciones entre sí, dan forma y reforman el sistema alimentario, o causan su inactividad o parálisis. Para comprender bien el sistema alimentario, resulta por lo tanto fundamental saber quiénes son los actores, cómo actúan e interactúan y por qué lo hacen. Por lo tanto, esta pieza comienza por definir quiénes son los actores y cuál es su ámbito de influencia. Rara vez el sistema alimentario permite jugar en igualdad de condiciones; la interacción entre los distintos actores se ve condicionada por los juegos de poder. Por lo tanto, recomendamos hacer un análisis de economía política y gobernanza en la etapa fina del análisis del sistema alimentario. Con ello, obtendremos una panorámica de las dinámicas de poder del sistema: quién se beneficia y quién queda excluido en los distintos ejes sociales de privilegio/marginación, lo que implica conocer la propia posición.

\section{Características del sistema}

Esta pieza tiene como objetivo ofrecer una visión general de los problemas a tratar y la situación actual de diversos elementos del sistema alimentario. El análisis del sistema alimentario trata de entender el problema que se haya definido en el objetivo; en otras palabras, descubrir las dinámicas de los resultados socioeconómicos., de seguridad alimentaria y de la nutrición y medioambientales.

Además de conocer los resultados del sistema alimentario esta pieza mapea sus distintos elementos: las actividades (ej.: la cadena de valor), los motores subyacentes y el contexto institucional y medioambiental. El mapeo incluye la evaluación de los indicadores clave, así como la comprensión de las tendencias (históricas), sinergias y equilibrios de actividades e indicadores concretos.

\section{Comportamiento del sistema}

Mientras que las características del sistema tienen que ver con la situación y las tendencias de diversos elementos del sistema alimentario, el comportamiento del sistema se ocupa de las interacciones entre los elementos. Por ejemplo, el impacto del cambio climático en las prácticas agrarias, o la influencia de las políticas sobre el comercio local. Un análisis del comportamiento del sistema resulta fundamental para entender el sistema alimentario y cómo se producen los cambios sistémicos; es decir cómo afecta el cambio en una parte del sistema a las demás.

Esta pieza examina concretamente las relaciones causales entre los distintos elementos del sistema alimentario. La identificación de los arquetipos del sistema (patrones comunes de comportamiento del sistema) hace posible entender mejor cómo se comporta e identificar palancas de cambio. Aunque recomendamos utilizar los arquetipos del sistema alimentario en su análisis, no es necesario hacerlo. Sin embargo, entender las relaciones causales en el seno del sistema es necesario para identificar palancas. Se trata de ámbitos en el seno del sistema alimentario en los que las intervenciones con un objetivo claro pueden cambiar sus resultados.

\section{Principios de calidad}

En todas las etapas del análisis del sistema alimentario hay tres principios de calidad que se deben tener en cuenta: pensamiento del sistema, implicación de los grupos de interés y atención a la equidad e inclusión. En los siguientes capítulos se profundiza en lo que significan y suponen estos principios de calidad. En las descripciones de las herramientas, existen guías para integrar los principios de calidad en el análisis. 


\section{Principio de calidad A: Pensamiento del sistema}

Un sistema alimentario es la interrelación dinámica entre muchos elementos: no se trata de una máquina estática que produce resultados en función de determinados parámetros de entrada. A la clase de cambios que nos gustaría ver en los sistemas alimentarios los llamamos cambios sistémicos. Sistémico quiere decir en relación con todo el sistema, y no es lo mismo que sistemático. Por lo tanto, en vez de dividir un sistema alimentario en elementos pequeños con el fin de analizarlos, lo analizamos en su totalidad, observando la relación entre sus elementos y cómo se comporta. Eso invita a analizar de una forma distinta, o a utilizar herramientas mentales diferentes, como las indicadas a continuación. Un análisis del sistema que también trata de entender aquellos ámbitos del mismo que no se pueden observar o cuantificar fácilmente. También entendemos un sistema como una realidad compleja en la que las relaciones causa-efecto solo aparecen cuando se toman medidas (la emergencia). Aprender desde dentro del sistema con varios grupos de interés es un requisito previo para conocer mejor el sistema (véase Posthumus et al., 2018a para conocer el marco conceptual con más detalle).

La intervención en el sistema tiene como objetivo modificar el statu quo de un ámbito problemático para llevarlo a un estado más deseable, que puede que exija intervención en algunos ámbitos no evidentes del sistema. También reconoce que es probable que haya resistencias al cambio en el seno del sistema que tengan que tratarse, entre grupos sociales concretos o en el seno de las actuales estructuras institucionales. Aunque se suele hablar de la transformación de los sistemas alimentarios no es posible desmantelar un sistema alimentario que ya existe y rediseñarlo desde cero. Las intervenciones en el sistema tratan, por tanto, de empujar a los sistemas alimentarios en la dirección que se quiere que vayan, para

Herramientas de un pensador del sistema (adaptado de Leyla Acaroglu/Disruptive Design)

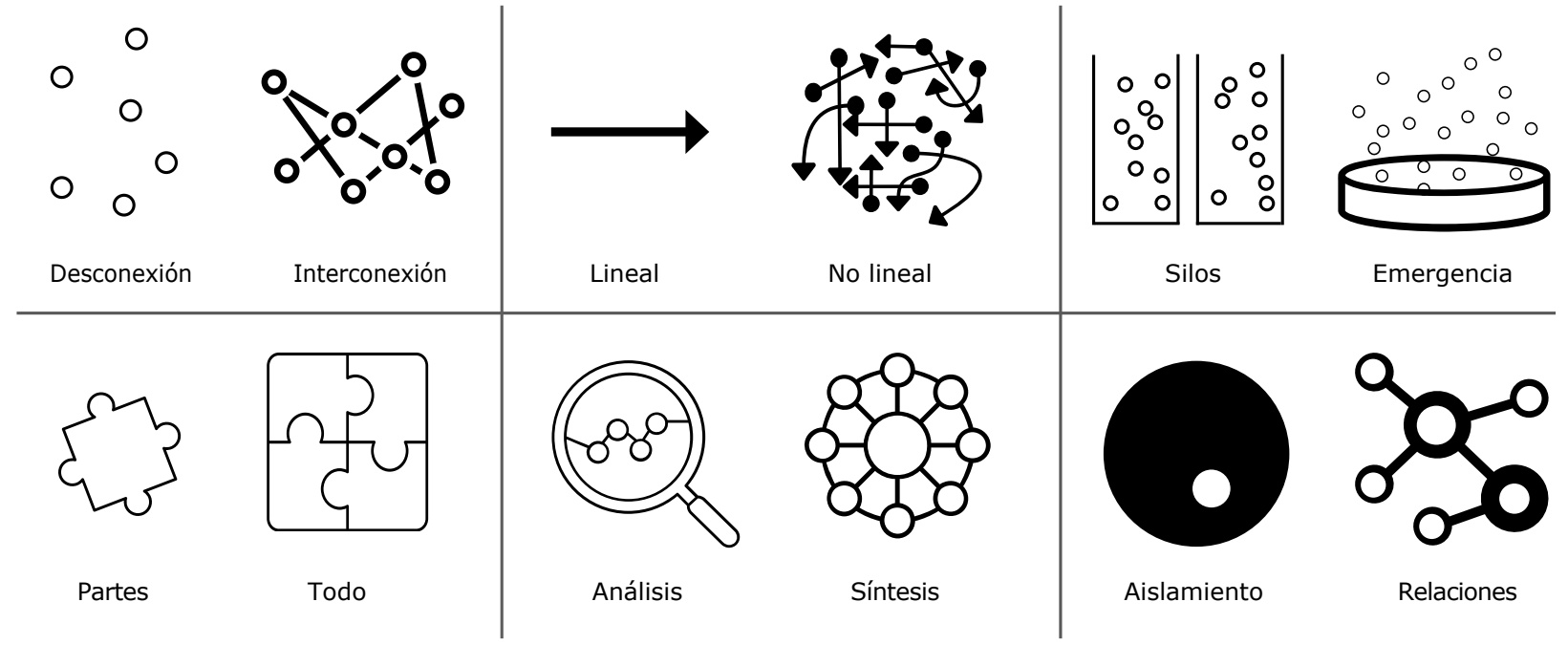

que abandonen el statu quo. Esto se lleva a cabo fijando como objetivo palancas que pueden hacer que el sistema avance hacia un estado más deseable, más sostenible para la salud humana y del planeta. Se entiende por palanca aquel lugar dentro de un sistema complejo en el que un pequeño cambio en un factor o proceso puede generar un impacto de mucho calado sobre el sistema alimentario (véase, p. ej.: Meadows, 1999).

\section{Su guía de bolsillo en el pensamiento del} sistema

1. Céntrese dentro de los límites de su sistema; evite enmarañarse en la red de todo el cosmos.

2. Busque patrones en el comportamiento que el sistema haya tenido en el pasado.

3. Estudie las relaciones entre los distintos elementos de su sistema.

4. Trate de entender el sistema, no pretenda arreglarlo; así es como surgen las soluciones.

5. No arrastre al sistema a una solución estática; vaya empujándolo poco a poco en la dirección que quiera que avance.

6. Acepte la ambigüedad

7. Sea flexible con sus ideas, pensamientos y procesos; déjelos evolucionar.

8. Acérquese y aléjese reiteradamente del sistema cabalgando el funcionamiento del sistema a nivel micro y macro y sus elementos

9. No existe una solución mágica que pueda arreglar e sistema; trate de entender cómo afecta cada una de ellas, incluidos los efectos negativos no deseados en relación con otras.

Extraido de: Rockefeller 2019, p31 


\section{Principio de calidad B: Implicación de los grupos de interés}

\section{¿Por qué es importante implicar a los grupos de interés?}

Al analizar un sistema alimentario, puede ser difícil decidir quién queremos que participe en el análisis. Hay muchas opiniones distintas y contradictorias entre los distintos grupos de interés en cuanto al funcionamiento del sistema alimentario. No obstante, resulta fundamental incluir estas voces diversas en su análisis, por dos motivos: a) ofrece más perspectivas a tener en cuenta sobre el sistema alimentario, por lo que hay más posibilidades para que surjan innovaciones nuevas; $b$ ) invita a los grupos de interés a entrar en el debate sobre el cambio del sistema alimentario para conseguir mejores resultados para todos y crear una perspectiva práctica al mismo tiempo.

\section{Participaciones y grupos de interés: ¿quiénes son} los grupos de interés?

Todos aquellos que tengan intereses en el sistema alimentario, o a quienes les preocupe, son grupos de interés. En otras palabras, cualquier grupo o persona que pueda afectar al sistema alimentario o a quien este le afecte. De cara a su análisis, es importante reconocer sus motivaciones, activos, preocupaciones y agendas. Sin embargo, tampoco resulta práctico que absolutamente todos estén representados. La etapa Definición de los actores y su ámbito de influencia ofrece varias formas de analizar y priorizar los grupos de interés que deben participar en su análisis.

Asegúrese de contar con representación de todos los principales grupos de interés: administraciones públicas, sector privado, sociedad civil (ej.: organizaciones agrarias, organizaciones comunitarias, ONG) e instituciones dedicadas a la difusión del conocimiento. Trate además de identificar a grupos de interés más allá de aquellos en los que normalmente se pensaría para escuchar voces que normalmente no se escuchan en esta etapa (por ejemplo, grupos de mujeres). Puede incorporar grupos o clústeres que operen a niveles distintos, en función del contexto o el tamaño de su análisis. Entre estos grupos, trate de hacer todo lo posible por contar con una selección transversal de las distintas categorías sociales (p. ej.: género, edad, nivel de renta). Tenga en cuenta que no todos los grupos de interés tienen los mismos problemas. Es posible que uno de ellos tenga un problema concreto, que los demás no tienen necesariamente que compartir. Además, sea consciente de sus propios sesgos a la hora de elegir a los grupos de interés.

\section{Organizar a sus grupos de interés: talleres, grupos} de debate y entrevistas

No todos los grupos de interés tienen tiempo de participa en un proceso de análisis ni les interesa hacerlo. Eso no quiere decir que no debería implicarles. En general, se recomienda organizar un taller con los grupos de interés para intercambiar perspectivas y conocer los puntos en los que los participantes están de acuerdo en desacuerdo. No obstante, puede que esto no sirva para todos. Piense en los legisladores de alto nivel que no pueden estar todo un día en un taller (consejo: infórmeles antes del taller, entrevístese con ellos si es posible, y transmítales información después) o en los miembros de la comunidad que no se sienten cómodos en un taller (consejo: ayúdeles a contar su historia en vídeo o a través de internet, $y$ pídales permiso para utilizar su testimonio en el taller). Si no es factible o deseable organizar un taller, utilice las deliberaciones de grupos de debate o las entrevistas semiestructuradas para recopilar las perspectivas de los distintos grupos de interés sobre el sistema alimentario. Trate de crear un pequeño equipo de validación con varios grupos de interés que comente el análisis de los datos y lo enriquezca.

El objetivo no es implicar a todos todo el tiempo, sino hacer un análisis que tenga en cuenta las perspectivas de los distintos grupos de interés, incorpore distintos tipos de conocimiento y facilite las oportunidades para crear un mayor entendimiento y más conexiones entre ellos. Un análisis del sistema alimentario puede por lo tanto también capacitar a los grupos de interés para iniciar acciones con el fin de dar forma a los sistemas alimentarios conjuntamente y ofrecer mejores resultados.

\section{Sea consciente de las dinámicas de poder, también} de su propia posición

Tenga en cuenta que los grupos de interés y sus relaciones no son neutras, y eso influye en sus (re)acciones. El momento y el lugar de la interacción es importante, puesto que puede excluir/incluir la asistencia de distintos tipos de grupos de interés (ej.: restricciones a la movilidad en función del sexo en función de la hora). Piense además en la disposición, la cultura local y el modo de facilitación para permitir que todas las voces se escuchen. Existen guías útiles en cuanto a los procesos con varios grupos de interés que ofrecen herramientas para lidiar con las dinámicas de poder en los talleres (ej.: La guía de MSP). Es importante recurrir a un facilitador neutral, altamente competente y de confianza, para que maneje las relaciones de poder complejas durante el taller. El apartado Análisis de economía política y gobernanza pone a su disposición varias herramientas para analizar las relaciones de poder dentro del sistema alimentario. 


\section{Principio de calidad C: Equidad e inclusión}

\section{En el seno de los sistemas alimentarios, los proce- sos funcionan de forma diferente para cada grupo}

Existen diferencias en las oportunidades y los desafíos entre los distintos grupos sociales si se tienen en cuenta una serie de características sociales (como sexo, edad, formación, profesión, clase social, discapacidad o pertenencia a minorías). No todo el mundo tiene el mismo acceso a los recursos ni puede beneficiarse en la misma medida de los resultados del sistema alimentario. Las desigualdades del sistema alimentario también se materializan de forma distinta en función de la etapa de la vida de una persona. Esta diversidad en el seno de los sistemas alimentarios en función de los diferentes grupos sociales se suele pasar por alto. Incluir los principios de equidad e inclusión en todas las etapas del análisis del sistema alimentario es clave para lograr un análisis de buena calidad. Pensar en la igualdad y el empoderamiento tiene mucho potencial de cara al análisis del sistema alimentario. En primer lugar, puede aclarar las causas raíz de la desigualdad en cuanto a los resultados del sistema alimentario para distintos grupos sociales y a distintos niveles. En segundo lugar, ofrece perspectivas sobre cómo afecta el poder a los procesos que contribuyen a la exclusión social y la marginación, que actúan como cuellos de botella estructurales con respecto a la igualdad (Newton, 2020).

\section{Empiece por identificar al grupo objetivo} pertinente

Las dinámicas del sistema alimentario se materializan de forma distinta dependiendo de quién sea cada uno, el acceso que tenga a recursos clave, las decisiones que pueda tomar y cómo afecten las costumbres sociales o de género al trabajo que pueda hacer o la capacidad de verse beneficiado por las intervenciones. Por lo tanto, es fundamental identificar a los grupos sociales más relevantes para los objetivos que se hayan propuesto. La edad y el género suelen ser factores importantes, pero piense también en otros marcadores

sociales intersecantes, como la religión, la sexualidad, la clase social, la raza, la condición de migrante, la renta, etc. En lo que respecta a los sistemas alimentarios, también resulta relevante distinguir dónde operan los actores de la cadena de suministro (ya sean proveedores de materias primas, agricultores a pequeña escala, productores comerciales, comerciantes, distribuidores, reguladores 0 consumidores), y cuánto poder tienen.

\section{Recapitulando: vaya más allá de la media}

os estudios suelen calcular medias. Por ejemplo, el valor nutricional medio de la dieta de una población. Sin embargo, las medias esconden diferencias sociales. Un desglose de los resultados del sistema alimentario según distintos marcadores sociales indicará quién necesita ayuda y de qué tipo. Esto supone buscar a personas y grupos desfavorecidos tanto sociocultural como nutricionalmente con motivo de los distintos factores que llevan a la malnutrición.

A lo largo del análisis del sistema alimentario, pregúntese habitualmente cómo difiere esto en función de quién sea cada uno, en función del sexo, la edad, la condición socioeconómica, la religión, la ubicación geográfica, etc.

Herramientas para incluir la igualdad y la inclusión en el análisis del sistema alimentario

Un paseo por la seguridad alimentaria

Esta herramienta aumenta la concienciación sobre los mecanismos de a desigualdad en el sistema alimentario. En función de la descripción de as características, los participantes responden a afirmaciones que les ayudan a tomar conciencia de cómo varían los resultados del sistema alimentario entre los diferentes grupos sociales.

Descubriendo los supuestos de género

Esta herramienta ofrece claridad sobre los supuestos de género, incluida la Teoría del Cambio, que a menudo no se ven, pero que influyen mucho. Se ocupa del riesgo de ignorar el género para lograr resultados de sistema alimentario inclusivos.
Ámbito, beneficio o empoderamiento

No todas las intervenciones 0 estrategias dirigidas a grupos desfavorecidos pretenden lograr el mismo resultado. Esta herramienta ayuda a entender la diferencia entre legar, beneficiar y empoderar a distintos grupos sociales. 


\section{Herramienta 1: Actores del sistema}

El funcionamiento de un sistema alimentario depende en gran medida de los actores que están presentes en él. Los actores del sistema incluyen a todas las personas, grupos y organizaciones que, de una forma u otra, están presentes en el sistema alimentario. Con sus interacciones,

construyen, reforman y ajustan el sistema alimentario. Para comprender bien el sistema alimentario, resulta por lo tanto fundamental saber quiénes son los actores, cómo actúan e interactúan y por qué lo hacen. Esto incluye tanto a los actores directamente implicados en las actividades relacionadas con la cadena de suministro de la alimentación, además de respaldar a los actores que prestan servicios en el seno del sistema alimentario (Figura 1). Por lo tanto, esta pieza comienza por definir quiénes son los actores y cuál es su ámbito de influencia.

Para entender aún mejor el sistema, también recomendamos hacer un análisis de economía política y gobernanza. Con ello, obtendremos una panorámica de las dinámicas de poder y los compromisos de gobernanza del sistema: quién se beneficia y quién queda excluido en los distintos ejes sociales de privilegio/marginación, lo que implica conocer la propia posición. Este análisis del poder y la gobernanza se debe hacer en la fase final del análisis global.

Un análisis de los actores del sistema debería hacerse preferiblemente a lo largo del análisis general: al principio para decidir a quién implicar en el análisis, en el transcurso del análisis para entender las diferencias en los beneficios, la influencia y los intereses entre cada uno de los grupos de interés de distintas áreas del sistema alimentario, y a fin de cuentas, saber cómo colaborar para activar las palancas y con quién hacerlo. En última instancia, esto fundamentará su estrategia con respecto al sistema alimentario.

\section{Esta pieza de la herramienta tiene dos partes:}

a. Definición de los actores y su ámbito de influencia

b. Análisis de economía política y gobernanza

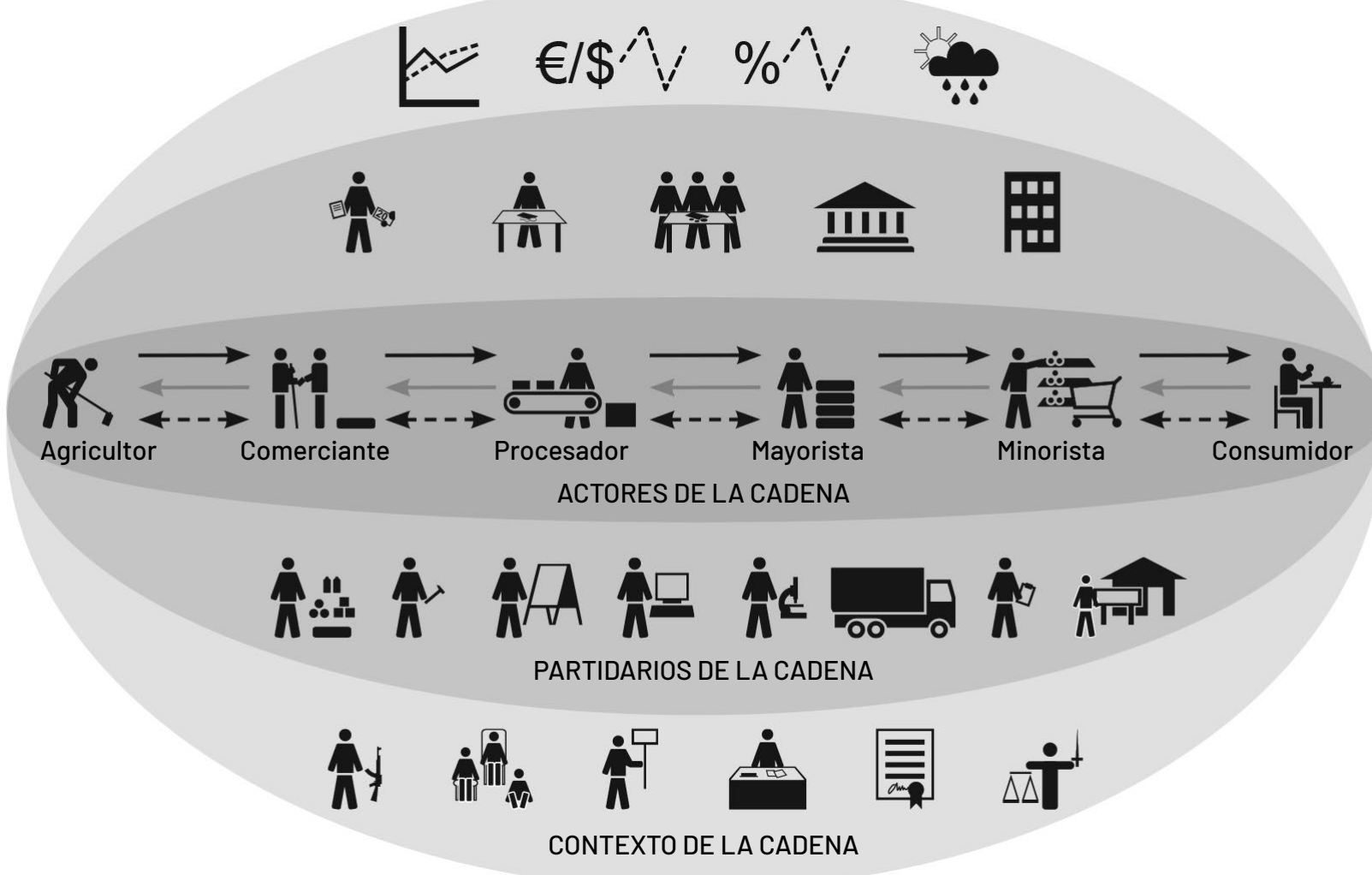




\section{Herramienta 1a: Definición de los actores y su ámbito de influencia}

\section{Introducción}

Estas herramientas ayudan a entender los roles que los distintos actores juegan en el sistema alimentario, sus objetivos y su ámbito de influencia. Las dinámicas del sistema son el resultado de los intereses y la influencia de los distintos actores, que se traducen en sus acciones e interacciones. Un análisis de los grupos de interés ofrece perspectivas sobre los intereses, mandatos, relaciones y niveles de poder de los distintos actores. Entender el campo de los grupos de interés, y saber quién puede activar las palancas de cambio, puede servir para fundamentar las estrategias de implicación de los distintos actores con el fin de lograr el objetivo que se haya formulado.

\section{Breve descripción en las herramientas}

Las herramientas ayudarán a adquirir un conocimiento en profundidad y estructurado sobre los distintos actores/ grupos de interés y sus ámbitos de influencia. Puede identificar los diversos grados de importancia de los grupos de interés concretos, así como su grado de influencia y cuá sería su aportación.

Al crear una red social, obtendrá una panorámica visual de cómo los actores están conectados. Al combinarlo con una representación visual de los objetivos de los grupos de interés en forma de gráficos radiales, veremos un cuadro muy detallado de los actores del sistema alimentario. Los resultados de estos ejercicios son importantes para el análisis desde el punto de vista de la economía política y la gobernanza.

\section{Principios de calidad}

Trate de incluir un amplio número de actores en esta etapa. Su papel podría consistir en facilitar información o participar activamente en el análisis. Piense en los grupos de interés formales e informales, actores conservadores $y$ transformadores, actores con distintos niveles y formas de influencia, actores procedentes de distintos contextos y actores con distintos roles en la cadena de suministro alimentaria, la sociedad, la comunidad y los hogares. Algo fundamental a tener en cuenta es a quién se incluye y a quién se deja fuera. Para más información, véanse los capítulos sobre la implicación de los grupos de interés y sobre igualdad e inclusión. Recuerde que no todo el mundo necesita implicarse a la vez ni tiene que estar de acuerdo en todo. Trate más bien de buscar un equilibrio entre inclusión y eficiencia. Pregúntese en todo momento: "¿Qué valor añadido aporta incluir a este actor, en este momento?"

\section{(2) Herramientas de concienciación}

\begin{tabular}{|c|c|}
\hline $\begin{array}{l}\text { Identificación de los } \\
\text { grupos de interés }\end{array}$ & $\begin{array}{l}\text { Características y } \\
\text { roles de los } \\
\text { grupos de interés }\end{array}$ \\
\hline $\begin{array}{l}\text { Visual rápida de los } \\
\text { grupos de interés más } \\
\text { relevantes para la } \\
\text { cuestión que se ana- } \\
\text { liza, y sus relaciones. } \\
\text { Asegúrese de incluir } \\
\text { una amplia gama de } \\
\text { grupos de interés, tal y } \\
\text { como hemos explicado } \\
\text { en los principios de } \\
\text { calidad. }\end{array}$ & $\begin{array}{l}\text { Esta herramienta } \\
\text { define los roles de los } \\
\text { grupos de interés en el } \\
\text { sistema alimentario. } \\
\text { Junto con la matriz } \\
\text { de importancia e in- } \\
\text { fluencia, los resultados } \\
\text { permiten adoptar un } \\
\text { enfoque y una estra- } \\
\text { tegia especificos para } \\
\text { cada grupo de interés. }\end{array}$ \\
\hline
\end{tabular}

$888<0-60 \mathrm{~min}$

$\begin{aligned} & \text { Mapeo } \\ & \text { del trayecto } \\ & \text { Visualización del } \\ & \text { proceso que siguen } \\ & \text { distintos actores (en } \\ & \text { particular, su grupo } \\ & \text { objetivo) para lograr } \\ & \text { sus objetivos. }\end{aligned}$
8R: @1-2 horas

\section{$\therefore$ Herramientas de exploración}

\begin{tabular}{|c|c|c|}
\hline $\begin{array}{l}\text { Matriz de } \\
\text { importancia e } \\
\text { influencia } \\
\text { Esta herramienta } \\
\text { capta el grado de } \\
\text { influencia y el nivel de } \\
\text { importancia que cada } \\
\text { grupo de interés les da } \\
\text { a los problemas que } \\
\text { se estén tratando o los } \\
\text { posibles objetivos. }\end{array}$ & $\begin{array}{l}\text { Análisis del } \\
\text { campo de fuerzas } \\
\text { En un grupo pequeño, } \\
\text { mapee los grupos de } \\
\text { interés que apoyan, o } \\
\text { son contrarios, a una } \\
\text { meta u objetivo de la } \\
\text { política. Las fuerzas se } \\
\text { puntúan en función de } \\
\text { su magnitud }\end{array}$ & $\begin{array}{l}\text { Inmersión } \\
\text { a través del } \\
\text { acompañamiento } \\
\text { Sumérjase en las vidas } \\
\text { de quienes trabajan en } \\
\text { el sistema alimentario } \\
\text { acompañándolos un dia } \\
\text { y preguntándoles cómo } \\
\text { toman decisiones. }\end{array}$ \\
\hline sf (-) 60-90 min & ifs (2) 1-2 horas & $\mathscr{S}^{1 \text { día con cada actor }}$ \\
\hline
\end{tabular}

.ill Herramientas de análisis

\begin{tabular}{|c|c|c|}
\hline $\begin{array}{l}\text { Análisis de la } \\
\text { red social }\end{array}$ & $\begin{array}{l}\text { Gráfico radial } \\
\text { de los objetivos }\end{array}$ & $\begin{array}{l}\text { Marco de acuerdos de } \\
\text { gobernanza del siste- } \\
\text { ma alimentario }\end{array}$ \\
\hline $\begin{array}{l}\text { Elabore un mapa social } \\
\text { de los actores para co- } \\
\text { nocer una perspectiva } \\
\text { de los contratos de } \\
\text { los grupos de interés, } \\
\text { a quién se transmite } \\
\text { el conocimiento y } \\
\text { los recursos y quién } \\
\text { asume el papel de in- } \\
\text { termediario. Los datos } \\
\text { se obtienen a través de } \\
\text { encuestas. }\end{array}$ & $\begin{array}{l}\text { Defina los objetivos } \\
\text { por parte de los grupos } \\
\text { de interés del sistema } \\
\text { alimentario e inclúya- } \\
\text { los en un gráfico. Para } \\
\text { cada uno de los grupos } \\
\text { de interés, cree un grá- } \\
\text { fico y puntúe. Ofrece } \\
\text { una panorámica visual } \\
\text { del sistema alimentario }\end{array}$ & $\begin{array}{l}\text { Con el fin de diagnos- } \\
\text { ticar las fortalezas } \\
\text { y debilidades de los } \\
\text { acuerdos de gobernanza } \\
\text { del sistema alimentario, } \\
\text { este marco describe } 5 \\
\text { principios: encuadre de } \\
\text { problemas basados en } \\
\text { el sistema, estructuras } \\
\text { de ampliación de los } \\
\text { límites, adaptabilidad, } \\
\text { inclusividad, capacidad } \\
\text { transformadora. }\end{array}$ \\
\hline 8 () ת 5-10 dias & (2) $0,5-2$ dias & (2) $0,5-2$ dias \\
\hline
\end{tabular}




\section{Herramienta 1b: Análisis de economía política y gobernanza}

\section{Introducción}

Esta etapa ofrece un conocimiento sobre las fuentes de poder que influyen sobre el sistema alimentario, y debería llevarse a cabo en la parte final del análisis. Profundiza sobre el anterior paquete de actores y ámbitos de influencia. Asimismo, se ocupa de la relación entre la economía política, las estructuras de gobernanza y las características y el comportamiento del sistema. En muchas intervenciones en el sistema alimentario la posición de los actores dominantes y los paradigmas tienden a salir reforzados, lo que da como resultado el mantenimiento del statu quo, $y$, en algunos casos, incluso se aumentan las desigualdades que ya existen. Por lo tanto, entender bien las dinámicas de poder, la política y la agencia en los sistemas alimentarios es fundamental para afrontar los problemas de una forma adecuada. Una vez terminada esta etapa, obtendrá una panorámica de las dinámicas de poder del sistema, quién se beneficia y quién queda excluido en los distintos ejes sociales de privilegio/ marginación, lo que implica conocer la propia posición. Esto dará como resultado una visión sobre los grupos de interés que podrían ser relevantes a la hora de influir sobre las palancas de cambio identificadas.

\section{Breve descripción en las herramientas}

El objetivo de las herramientas es entender qué formas de poder entran en juego y cómo la economía política y las estructuras de gobernanza interfieren con el sistema alimentario. Recomendamos empezar con las herramientas de concienciación que aumenten la sensibilización sobre las distintas formas de poder en el seno del sistema alimentario y quién se ve favorecido por el actual statu quo. Como siguiente paso, recomendamos analizar la economía política y administrativa vinculada al sistema alimentario, lo que incluye los marcos de política (alimentaria) locales. En la última parte del análisis, debe analizar cómo se distribuye el poder entre los distintos actores e instituciones en el seno del sistema sanitario y cómo influyen en el comportamiento del sistema, a través de las reglas del juego escritas y tácitas.

\section{Principios de calidad}

En este análisis, es fundamental ser sensible a las dinámicas de poder y a lo que se juega cada uno de los grupos de interés a los que invita a participar en talleres y entrevistas. Nadie es neutral. Eso incluye también su posición en calidad de investigador o solicitante del análisis del sistema alimentario. Para más información, consulte los capítulos sobre la implicación de los grupos de interés y sobre igualdad e inclusión.

\begin{tabular}{|c|c|c|}
\hline \multicolumn{3}{|c|}{ (2) Herramientas de concienciación } \\
\hline $\begin{array}{l}\text { Formas de poder } \\
\text { Esta herramienta ayu- } \\
\text { da a los participantes } \\
\text { a valorar qué formas } \\
\text { de poder se aplican en } \\
\text { el sistema alimenta- } \\
\text { rio. ¿Cómo tratan los } \\
\text { actores de influir en } \\
\text { el sistema? ¿Quieren } \\
\text { mantener el statu quo o } \\
\text { pretenden que haya un } \\
\text { cambio? }\end{array}$ & $\begin{array}{l}\text { Clasificación de } \\
\text { poder } \\
\text { Esta herramienta logra } \\
\text { que los participantes } \\
\text { se den cuenta de que } \\
\text { todas las personas } \\
\text { tienen situaciones } \\
\text { diferentes y disfrutan } \\
\text { de privilegios que pue- } \\
\text { den ser situacionales, } \\
\text { sociales y personales. } \\
\text { Estos atributos ofre- } \\
\text { cen al actor un cierto } \\
\text { nivel de poder. }\end{array}$ & $\begin{array}{l}\text { Indicadores de } \\
\text { gobernanza } \\
\text { Este conjunto de datos } \\
\text { global muestra una } \\
\text { serie histórica de seis } \\
\text { indicadores nacionales } \\
\text { de gobernanza (desde } \\
\text { 1996) relativos a } \\
\text { dimensiones como } \\
\text { la sostenibilidad, la } \\
\text { eficacia de la Adminis- } \\
\text { tración y la legislación } \\
\text { y regulación. }\end{array}$ \\
\hline 88845 & 8R8 1 hora & (-)1hora \\
\hline
\end{tabular}

\begin{tabular}{|c|c|c|c|c|c|}
\hline \multicolumn{3}{|c|}{ OO Herramientas de exploración } & \multicolumn{3}{|c|}{ III Herramientas de análisis } \\
\hline $\begin{array}{l}\text { El poder de los } \\
\text { grupos de interés } \\
\text { en los sistemas } \\
\text { alimentarios } \\
\text { Esta herramienta } \\
\text { ofrece una mayor com- } \\
\text { prensión del rol de los } \\
\text { grupos de interés en el } \\
\text { sistema alimentario. } \\
\text { Visualiza la impor- } \\
\text { tancia e influencia } \\
\text { relativa de cada uno de } \\
\text { los grupos de interés, } \\
\text { asi como su lugar en } \\
\text { el seno del sistema } \\
\text { alimentario }\end{array}$ & $\begin{array}{l}\text { Evaluación de la } \\
\text { economía política } \\
\text { Este método cua- } \\
\text { litativo explora el } \\
\text { compromiso político y } \\
\text { las oportunidades de } \\
\text { avanzar en las politicas } \\
\text { agroalimentarias. } \\
\text { Aúna el análisis de } \\
\text { los grupos de interés } \\
\text { con indicadores sobre } \\
\text { compromiso politico y } \\
\text { reformas. }\end{array}$ & $\begin{array}{l}\text { Las cinco A: } \\
\text { implicaciones } \\
\text { políticas } \\
\text { Tras evaluar la eco- } \\
\text { nomía politica, esta } \\
\text { herramienta ayuda a } \\
\text { identificar las impli- } \\
\text { caciones politicas: } \\
\text { ¿deberiamos tratar } \\
\text { de Alterar, Adaptar, } \\
\text { Ahorrar, Anhelar o } \\
\text { Abandonar prácticas/ } \\
\text { incentivos? }\end{array}$ & $\begin{array}{l}\text { Mapeo de red } \\
\text { Esta herramienta } \\
\text { combina el análisis de } \\
\text { red con el mapeo de } \\
\text { poder de los grupos de } \\
\text { interés y cómo influyen } \\
\text { sobre los resultados. } \\
\text { Indica en qué puntos } \\
\text { de la red se encuen- } \\
\text { tran las sinergias y los } \\
\text { conflictos entre grupos } \\
\text { de interés. }\end{array}$ & $\begin{array}{l}\text { Poder y } \\
\text { comportamiento del } \\
\text { sistema } \\
\text { Entrevistas sobre los } \\
\text { siguientes asuntos: } \\
\text { - ¿Cómo influyen los } \\
\text { actores y las estruc- } \\
\text { turas de gobernanza } \\
\text { sobre los circuitos de } \\
\text { retroalimentación? } \\
\text { - ¿Qué actores pueden } \\
\text { influir sobre las } \\
\text { palancas de cambio } \\
\text { identificadas? } \\
\text { - ¿Cuáles son sus } \\
\text { intereses? }\end{array}$ & $\begin{array}{l}\text { Futuros } \\
\text { escenarios } \\
\text { Con esta herramienta, } \\
\text { puede combinar los } \\
\text { cambios predecibles y } \\
\text { los no predecibles en el } \\
\text { sistema alimentario y } \\
\text { las dinámicas de poder } \\
\text { para esbozar posibles } \\
\text { resultados futuros. }\end{array}$ \\
\hline $898 \bumpeq ゚ 1-3$ días & $892 \mathfrak{R}^{1-3}$ dias & 898 @ $0,5-2$ dias & $888 \mathrm{~s}^{30-120 \mathrm{~min}}$ & $898 \mathfrak{\Omega}^{1-3}$ dias & (-) 1-5 dias \\
\hline
\end{tabular}




\section{Herramienta 2: Características del sistema}

Esta pieza analítica ofrece una panorámica del sistema alimentario, incluidos los resultados, actividades y motores subyacentes del sistema. Un análisis del sistema alimentario empieza por explorar los resultados del sistema alimentario. Querrá obtener perspectivas sobre qué genera realmente el sistema alimentario. Algunos ejemplos de resultados son una dieta saludable, la contaminación medioambiental y los sueldos dignos para los trabajadores agrícolas. No necesita analizar todos los resultados. Deje que su objetivo para el sistema alimentario (Parte 1) determine cuál será el foco de esta etapa de análisis.

Asimismo, se mapean los estados y las tendencias de los distintos elementos del sistema sanitario. El mapeo incluye la evaluación de los indicadores clave, así como la comprensión de las tendencias (históricas), sinergias y equilibrios entre distintas partes del sistema. Y, puesto que los sistemas alimentarios están conectados con muchos otros sistemas, están plagados de equilibrios, por lo que su transición hacia un modelo más sostenible se complica. El marco del sistema alimentario de van Berkum et al. (2018) puede utilizarse como una lista de comprobación de los temas que deben tratarse (Figura 2). De este modo, la atención se centrará en las vulnerabilidades del sistema alimentario, y se identificarán los factores más limitantes a la hora de lograr los resultados del sistema alimentario deseados.

En función de la disponibilidad de datos y las brechas de conocimiento, la pieza del análisis puede consistir en una revisión somera de los documentos y el recurso a los conocimientos expertos o enfoques más completos, como los estudios de ámbito y las encuestas para recopilar datos esenciales.
Figura 2. Marco del sistema alimentario de van Berkum et al. (2018)

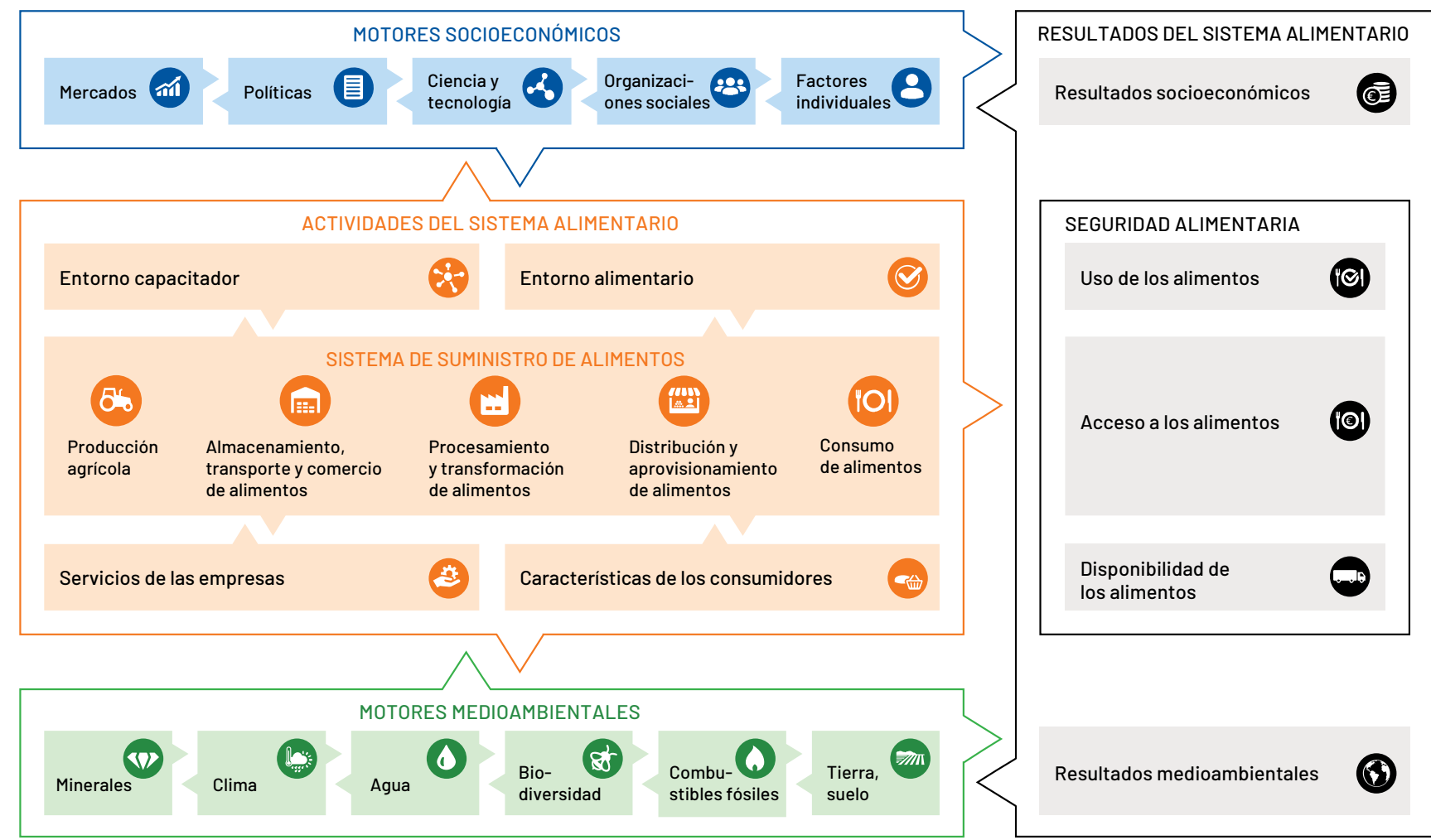

\section{Esta pieza del análisis tiene dos partes:}

a. Análisis de resultados del sistema alimentario

b. Mapeo de los motores y actividades del sistema alimentario 


\section{Herramienta 2a: Análisis de resultados del sistema alimentario}

\section{Introducción}

Los sistemas alimentarios producen tres tipos de resultados: de alimentación y nutrición, socioeconómicos y medioambientales. Aunque los resultados de alimentación y la nutrición de un sistema alimentario son los más importantes, se entiende los socioeconómicos y medioambientales también lo son en la misma medida.

\section{Breve descripción en las herramientas}

Para cada uno de los tres resultados, hemos incluido tres herramientas que pueden servirle para empezar, dependiendo de si lo que hace es aumentar la concienciación en el seno de un grupo, explorando el tema o analizándolo en más profundidad.

Si tiene la oportunidad de intervenir en un taller con varios grupos de interés, lo mejor es comenzar con una herramienta de concienciación. Buena parte de la exploración y el análisis más en profundidad puede hacerse en el seno de un grupo de expertos más pequeños.

\section{Principios de calidad}

Entender las diferencias en los resultados entre los distintos grupos sociales es fundamental para el análisis. Por ejemplo, cómo varía la dieta local entre hombres y mujeres, o entre los jóvenes y los mayores, y así con otros marcadores sociales. ¿Se deben esas diferencias a preferencias del consumidor, al acceso a los alimentos o a las dinámicas internas de los hogares? En el caso del análisis de gabinete, como el de la brecha nutricional, es importante mirar más allá de las medias y desglosar los resultados en función de los distintos grupos sociales.

\begin{tabular}{|c|c|c|c|c|c|c|c|c|}
\hline \multicolumn{3}{|c|}{ (O) Herramientas de concienciación } & \multicolumn{3}{|c|}{ OO Herramientas de exploración } & \multicolumn{3}{|c|}{ IIl Herramientas de análisis } \\
\hline $\begin{array}{l}\text { De alimentación y } \\
\text { nutrición }\end{array}$ & Socioeconómicos & Medioambientales & $\begin{array}{l}\text { De alimentación y } \\
\text { nutrición }\end{array}$ & Socioeconómicos & Medioambientales & $\begin{array}{l}\text { De alimentación y } \\
\text { nutrición }\end{array}$ & Socioeconómicos & Medioambientales \\
\hline $\begin{array}{l}\text { Lo que comemos } \\
\text { Dibuje un plato y } \\
\text { dividalo en función } \\
\text { de los ingredientes/ } \\
\text { grupos de alimentos } \\
\text { más tipicos que se } \\
\text { consumen en la dieta } \\
\text { local, y sus lugares de } \\
\text { procedencia. }\end{array}$ & $\begin{array}{l}\text { Clasificación de la } \\
\text { riqueza } \\
\text { Herramienta de } \\
\text { participación para } \\
\text { identificar las clases } \\
\text { de riqueza y sus } \\
\text { atributos en el seno de } \\
\text { las sociedades. }\end{array}$ & $\begin{array}{l}\text { Un paseo } \\
\text { transversal } \\
\text { Organice un paseo } \\
\text { guiado a través de un } \\
\text { paisaje para lograr una } \\
\text { visión integral de cómo } \\
\text { conviven y/o compiten } \\
\text { la agricultura, } \\
\text { la naturalezay } \\
\text { las actividades } \\
\text { económicas. } \\
\text { l0,5 dias }\end{array}$ & $\begin{array}{l}\text { Coste mínimo de } \\
\text { una dieta saludable } \\
\text { Método de cálculo en } \\
\text { el que se combinan } \\
\text { los precios de } \\
\text { mercado y los valores } \\
\text { nutricionales de las } \\
\text { cosechas locales. } \\
\text { Indica el coste de una } \\
\text { dieta sanay cuáles } \\
\text { son los productos } \\
\text { alimentarios con } \\
\text { buena relación calidad/ } \\
\text { precio. }\end{array}$ & $\begin{array}{l}\text { Evaluación rápida } \\
\text { del sector } \\
\text { Esta evaluación } \\
\text { ayuda a profundizar } \\
\text { en la evaluación de } \\
\text { un sector, a través } \\
\text { de un modelo de } \\
\text { Transformación } \\
\text { Sectorial. Combina } \\
\text { las encuestas con } \\
\text { las deliberaciones de } \\
\text { grupos de debate. Este } \\
\text { ejemplo versa sobre el } \\
\text { impacto de la CoVID-19 } \\
\text { en la agricultura. }\end{array}$ & $\begin{array}{l}\text { Mapeo del uso } \\
\text { participativo de la } \\
\text { tierra } \\
\text { Indica cómo se } \\
\text { distribuye el uso de } \\
\text { la tierra y cómo ha } \\
\text { cambiado a lo largo del } \\
\text { tiempo. Muestra las } \\
\text { perspectivas locales en } \\
\text { cuanto a los recursos } \\
\text { naturales, asi como } \\
\text { dónde se encuentran } \\
\text { las vulnerabilidades. }\end{array}$ & $\begin{array}{l}\text { Análisis de la brecha } \\
\text { nutricional } \\
\text { En función de los datos } \\
\text { sobre disponibilidad } \\
\text { de alimentos y salud, } \\
\text { puede analizar qué } \\
\text { elementos faltan en } \\
\text { las dietas, también en } \\
\text { función de diversos } \\
\text { grupos objetivo. Una } \\
\text { buena fuente es el } \\
\text { programa DHS. }\end{array}$ & \multicolumn{2}{|c|}{$\begin{array}{l}\text { Análisis donut } \\
\text { Utilice el modelo donut de Kate Raworth para } \\
\text { encontrar el punto idóneo para que los sistemas } \\
\text { alimentarios respalden unas vidas saludables sin } \\
\text { necesidad de salir de nuestro planeta. Consulte } \\
\text { también el Good Life Index. } \\
\text { Se puede aplicar a países, paisajes, ciudades, etc }\end{array}$} \\
\hline is? 1 hora & 892 horas & $8980,5-2$ dias & (2) 1-3 dias & 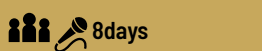 & $8890,5-2$ días & (2) 2-10 dias & \multicolumn{2}{|l|}{ (อ):3: 1-5 dias } \\
\hline
\end{tabular}




\section{Herramienta 2b: Mapeo de los motores y actividades del sistema alimentario}

\section{Introducción}

Tendrá que profundizar en las diversas actividades que tienen lugar en un sistema alimentario y tener claro qué las impulsa (son las partes naranjas, azules y verdes del modelo del sistema alimentario). Debe centrarse en las tendencias que pueda identificar a través de indicadores clave. Los motores de las actividades del sistema alimentario pueden por tanto considerarse la base del análisis. Recuerde que un mapeo es siempre una foto fija, mientras que, en realidad, los elementos del sistema alimentario son dinámicos.

\section{Breve descripción en las herramientas}

Las herramientas en esta etapa son por naturaleza más genéricas, pero son fundamentales para conocer los motores y tendencias de los sistemas alimentarios. Para centrar el análisis de las tendencias del sistema, trate de centrarse en aquellos aspectos que estén más relacionados con el objetivo que se haya formulado. Asegúrese, no obstante, de que el análisis incluye los aspectos de todos los motores y resultados, con el fin de lograr una visión general del sistema.

Recomendamos empezar con entrevistas, una revisión de la literatura publicada, la recopilación de datos generales y la triangulación sobre estas fuentes para generar una primera panorámica. El nivel de esfuerzo necesario depende del conocimiento previo del sistema alimentario que nos interese. Tenga en cuenta que hay muchas fuentes secundarias de datos disponibles de forma gratuita. Para profundizar en el análisis, recomendamos algunas herramientas de análisis para entender las sinergias y equilibrios que generan algunas tendencias y cómo podrian evolucionar las tendencias en el futuro.

\section{Principios de calidad}

Es importante evitar que los datos que recopile estén sesgados. Así pues, asegúrese de que los informantes y la literatura claves representen a los grupos objetivo pertinentes. En los mercados emergentes, buena parte del sistema alimentario se enmarca en la economía sumergida, por lo que el análisis debería, por tanto, incluir los mercados, actividades y flujos comerciales no registrados.

Con la recopilación de datos, es necesario prestar atención a cuestiones parecidas. Asegúrese de que el análisis va más allá del cálculo de la media. Incluya en él también a los grupos objetivo pertinentes y analice las diferencias entre ellos. Para más detalles, véase el capítulo sobre igualdad e inclusión.

\section{O Herramientas de concienciación}

\begin{tabular}{|c|c|c|}
\hline Entrevistas & Mapa interactivo & Datos secundarios \\
\hline $\begin{array}{l}\text { Entrevistas para } \\
\text { entender qué } \\
\text { consideran los } \\
\text { informantes clave } \\
\text { tendencias, motores y } \\
\text { actividades clave del } \\
\text { sistema alimentario. }\end{array}$ & $\begin{array}{l}\text { Use Google Earth } \\
\text { (online o con impresión } \\
\text { a gran escala) en los } \\
\text { talleres o entrevistas } \\
\text { con los grupos de } \\
\text { interés. Pida a los } \\
\text { actores del sistema } \\
\text { alimentario que identi- } \\
\text { fiquen su papel en las } \\
\text { actividades que van } \\
\text { desde la producción al } \\
\text { consumo. Añada datos } \\
\text { al mapa (ejemplo, } \\
\text { Kenia/Bolivia). }\end{array}$ & $\begin{array}{l}\text { Recopile datos secun- } \\
\text { darios en relación con } \\
\text { el sistema alimentario. } \\
\text { Algunos ejemplos } \\
\text { serian: datos de PIB, } \\
\text { agricultura, nutrición, } \\
\text { medioambiente y regu- } \\
\text { laciones comerciales. } \\
\text { Algunas fuentes fiables } \\
\text { son el Banco Mundial } \\
\text { (datos, facilidad para } \\
\text { hacer negocios) y el } \\
\text { Tablero de los Siste- } \\
\text { mas Alimentarios. }\end{array}$ \\
\hline 1-14 días & (2) $0,5-5$ dias & (ㅇ) $0,5-5$ dias \\
\hline
\end{tabular}

\section{$\therefore$ Herramientas de exploración}

Una cadena de valor que tiene en cuenta la nutrición

Esta herramienta identifica las inversiones de la cadena de valor que tienen en cuenta la nutrición. Recomendamos el tercer paso en la guía del FIDA como complemento al resto de herramientas que aparecen en esta guía.

\& ๑2-3 semanas

Herramienta
integrada de
evaluación de la
biodiversidad
Ayuda a comprobar
la situación de un
punto concreto
dentro del sistema
alimentario en cuanto
a la biodiversidad. El
primer nivel de acceso
es gratuito para todo el
mundo.

Revisión de la literatura publicada Una revisión de la literatura publicada sobre aquello que los principales autores describen como los principales motores $y$ tendencias del sistem alimentario.

(2) 0,5-5 dias

\section{.ili Herramientas de análisis}

\begin{tabular}{|c|c|c|}
\hline $\begin{array}{l}\text { Sinergias } \\
\text { y equilibrios }\end{array}$ & $\begin{array}{l}\text { Futuros } \\
\text { escenarios }\end{array}$ & $\begin{array}{l}\text { Análisis de las } \\
\text { políticas }\end{array}$ \\
\hline $\begin{array}{l}\text { Mapa de las tendencias } \\
\text { importantes del siste- } \\
\text { ma alimentario y yus } \\
\text { interacciones. ¿Cuáles } \\
\text { pueden fortalecer unas } \\
\text { a otras y cuáles crean } \\
\text { equilibrios? Sintesis de } \\
\text { ejemplo para que sirva } \\
\text { de inspiración. }\end{array}$ & $\begin{array}{l}\text { Esta herramienta } \\
\text { combina los cambios } \\
\text { predecibles y los no } \\
\text { predecibles en el } \\
\text { sistema alimentario } \\
\text { para esbozar } \\
\text { posibles resultados } \\
\text { futuros. Puede } \\
\text { encontrar algunos } \\
\text { buenos ejemplos en } \\
\text { Foresight4Food }\end{array}$ & $\begin{array}{l}\text { Analice las politicas } \\
\text { locales, nacionales e } \\
\text { internacionales que } \\
\text { afectan al sistema ali- } \\
\text { mentario. Entre ellas, } \\
\text { puede haber politicas } \\
\text { de las Administracio- } \\
\text { nes, pero también de } \\
\text { otras organizaciones } \\
\text { influyentes. Esta es } \\
\text { una guia práctica. }\end{array}$ \\
\hline (2) $1-5$ dias & (2) $>30$ días & 乞̊ (3-10 días \\
\hline
\end{tabular}




\section{Herramienta 3: Comportamiento del sistema}

El comportamiento del sistema se ocupa de las interacciones entre los elementos del sistema alimentario mientras que las características del sistema analizan la situación y las tendencias de los distintos elementos que lo componen. Por ejemplo, ¿cómo influye el cambio climático en las prácticas agrícolas? Y, ¿cómo influyen las políticas del gobierno en las estructuras del comercio local? El análisis del comportamiento del sistema es fundamental a la hora de entender el sistema alimentario y diseñar estrategias de intervención eficaces. Muestra cómo el cambio en una parte del sistema afecta al resto.

Esta pieza consta de tres partes. La primera consiste en identificar a las víctimas del sistema. Ofrece un diagrama visual de las relaciones causales entre los distintos

elementos del sistema alimentario. En segundo lugar, es posible etiquetar las diversas relaciones causales como arquetipos del sistema. Esto ayuda a entender patrones comunes de comportamiento que se repiten en el seno de los sistemas alimentarios. De este modo, es posible identificar las palancas para lograr un cambio sistémico. Aunque recomendamos utilizar los arquetipos del sistema alimentario en su análisis, no es necesario hacerlo. En último lugar, se deberían identificar las palancas de cambio del sistema. Se trata de ámbitos de las relaciones causales en los que las acciones pueden provocar un cambio del sistema, y, más tarde, un impacto valioso.

En el pensamiento de los sistemas, normalmente el sistema se representa como un iceberg del que el observador solo ve una pequeña parte (los acontecimientos y síntomas), pero que son el resultado de lo que ocurre bajo la superficie: los patrones, estructuras y paradigmas o modelos mentales que determinan el comportamiento del sistema (véase la Figura 3). El pensamiento en las palancas afirma que cuanto más se profundiza en el sistema con las intervenciones, más palanca para el cambio sistémico puede generarse (véase el cuadro sobre los puntos de intervención de Meadows).
Figura 3. El modelo del iceberg
Acontecimientos y síntomas ¿Qué ha pasado?

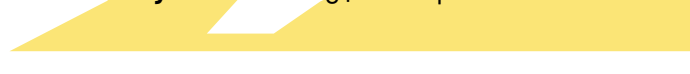

Estructura delsistema ¿Qué podría explicar ............. Diseñar los patrones/ acontecimientos?

Modelosmentales ¿En qué medida nuestro pensamiento permite que esta situación continúe?
Transformar

Reaccionar

Anticipar

\section{Palancas para el cambio sistémico. Intervenciones, de menor a mayor eficacia (Meadows, 1999):}

1. Cambiar cualquier cifra que se pueda medir: constantes, parámetros, números (subsidios, impuestos, normas)

2. Cambiar la forma en la que las existencias y los flujos se conectan: flujos sustanciales y nodos de intersección sustancial. y síntomas

Patrones

3. Ajuste las ratios de retraso en relación con la tasa de cambio sistémico.

4. Permitir que algo se ajuste más rápido a las metas: regulación de los circuitos de retroalimentación negativa.

5. Permitir que algo crezca más rápido: impulso de los circuitos de retroalimentación positiva.

Estructura del sistema

6. Mejorar los flujos de información.

7. Cambiar las reglas del sistema (incentivos, penalizaciones, restricciones).

8. Permitir diversas formas de autorregulación (ej.: intervención en la distribución de poder)

9. Cambiar las metas del sistema.

10. Cambiar el esquema mental o el paradigma del que surge el sistema. 


\section{Herramienta 3: Identificar a las víctimas del sistema}

\section{Introducción}

El Mapeo causal ofrece perspectivas sobre las causas raíz de las dinámicas del sistema alimentario. Los mapas causales ilustran cómo el cambio en un factor afecta al cambio en otro factor en una dirección parecida (positivo) o contraria (negativo). Los mapas causales también incluyen circuitos de retroalimentación entre distintos factores que pueden reforzar o restringir procesos concretos en el seno de un sistema. Identificar a las víctimas del sistema es un paso clave para analizar cómo los distintos motores y actividades pueden influir entre sí, y, posteriormente, sobre los resultados del sistema alimentario. Asegúrese de que aporta nuevas perspectivas al paso previo (Mapeo de los motores y actividades del sistema alimentario).

\section{Breve descripción en las herramientas}

En un entorno de taller, recomendamos para empezar hacer un ejercicio de árbol de los problemas con los grupos de interés pertinentes, con el fin de incorporar perspectivas locales sobre las causas y efectos en el sistema alimentario local. Si los grupos de interés tienen dificultades para elaborar este árbol de problemas, es posible empezar elaborando un mapa conceptual o gráfico $Q Q$ como tormenta de ideas en relación con problemas relativos al resultado del sistema alimentario de que se trate. Muchas de estas herramientas también pueden utilizarse a través de internet combinando videollamadas con, por ejemplo, plantillas de mural. Es posible recurrir a otras herramientas, como la creación de diagramas de circuito causal. En función del árbol de problemas, los analistas del sistema pueden profundizar más en los vínculos causales y la influencia de los circuitos de retroalimentación sobre e comportamiento del sistema en su conjunto. Con el fin de realizar la recopilación de datos por parte de encuestados se puede utilizar un software que tenga en cuenta el sistema, como Sensemaker. Este análisis revela los procesos no lineales que determinan el comportamiento del sistema alimentario y sus resultados, y es importante de cara a la identificación de los arquetipos del sistema.

\section{Principios de calidad}

Las relaciones causales del sistema alimentario pueden materializarse de forma distinta en los diferentes grupos sociales. Por tanto, se pueden elaborar distintos árboles de problemas para distintos grupos objetivo. Para hacerlo de forma eficaz, asegúrese de que los participantes del taller incluyen representantes de los grupos. Las sesiones separadas para distintos grupos sociales también son una opción. En cuanto a la recopilación de datos, es necesario prestar atención a cuestiones similares. Asegúrese de que el análisis va más allá del cálculo de la media. Incluya en él también a los grupos objetivo pertinentes y analice las diferencias entre ellos. Para más detalles, véase el capítulo sobre igualdad e inclusión.

\begin{tabular}{|c|c|c|c|c|c|c|c|c|}
\hline \multicolumn{3}{|c|}{ (2) Herramientas de concienciación } & \multicolumn{3}{|c|}{ OO Herramientas de exploración } & \multicolumn{3}{|c|}{ - II Herramientas de análisis } \\
\hline $\begin{array}{l}\text { Mapa conceptual } \\
\text { Sesión de tormenta } \\
\text { de ideas, en la que } \\
\text { se elabora una } \\
\text { representación visual } \\
\text { de ideas e información } \\
\text { relacionadas del grupo } \\
\text { sobre el sistema } \\
\text { alimentario. }\end{array}$ & $\begin{array}{l}\text { Gráfico } Q Q \\
\text { El ejercicio de tor- } \\
\text { menta de ideas pide } \\
\text { a los participantes } \\
\text { que distingan entre } \\
\text { las variables duras } \\
\text { (cuantitativas)y blan- } \\
\text { das (cualitativas) que } \\
\text { afectan a una cuestión } \\
\text { concreta. Ayuda a los } \\
\text { participantes a obtener } \\
\text { una panorámica del } \\
\text { sistema. } \\
\text { \&\$ } 60-120 \text { min }\end{array}$ & $\begin{array}{l}\text { Diagrama de } \\
\text { afinidad } \\
\text { La sesión de tormenta } \\
\text { de ideas, en la que los } \\
\text { participantes anotan } \\
\text { sus propias ideas } \\
\text { en tarjetas que más } \\
\text { tarde el grupo clasifica } \\
\text { en grandes temas y } \\
\text { problemas. }\end{array}$ & $\begin{array}{l}\text { Árbol de problemas } \\
\text { Esta herramienta } \\
\text { se suele utilizar en } \\
\text { la planificación de } \\
\text { proyectos. Ofrece una } \\
\text { estructura que mapea } \\
\text { las causas raíces y } \\
\text { efectos de un problema } \\
\text { en particular. }\end{array}$ & $\begin{array}{l}\text { Comportamiento a } \\
\text { lo largo del tiempo } \\
\text { Indica cómo se } \\
\text { interrelacionan } \\
\text { las variables de } \\
\text { comportamiento. } \\
\text { Responde a preguntas } \\
\text { como: si ocurre esto } \\
\text { con una variable, ¿qué } \\
\text { les pasa a las demás? }\end{array}$ & $\begin{array}{l}\text { Diagrama de } \\
\text { función gráfica } \\
\text { Indica cómo se afectan } \\
\text { dos variables entre sí. } \\
\text { Esta relación se refleja } \\
\text { en un gráfico. También } \\
\text { ayuda a entender las } \\
\text { relaciones no lineales. }\end{array}$ & $\begin{array}{l}\text { Diagrama de } \\
\text { circuito causal } \\
\text { Este análisis revela los } \\
\text { procesos no lineales } \\
\text { que determinan el } \\
\text { comportamiento del } \\
\text { sistema alimentario } \\
\text { y sus resultados, y es } \\
\text { importante de cara a } \\
\text { la identificación de los } \\
\text { arquetipos del sistema. }\end{array}$ & $\begin{array}{l}\text { Modelos basados en } \\
\text { agentes } \\
\text { Técnica de los modelos } \\
\text { de simulación que } \\
\text { mapea los efectos de } \\
\text { la toma de decisiones } \\
\text { autónoma sobre } \\
\text { los patrones de } \\
\text { comportamiento } \\
\text { complejos en un } \\
\text { sistema alimentario. }\end{array}$ & $\begin{array}{l}\text { Sensemaker } \\
\text { Software de Cognitive } \\
\text { Edge utilizado para } \\
\text { recopilar datos, } \\
\text { recoger testimonios } \\
\text { de personas en su } \\
\text { contexto y hacer } \\
\text { posible el reconoci- } \\
\text { miento de patrones y } \\
\text { el descubrimiento de } \\
\text { situaciones distintas a } \\
\text { la tendencia. } \\
\\
\text { @- > } 30 \text { días }\end{array}$ \\
\hline
\end{tabular}




\section{Herramienta 3: Etiquetado de arquetipos del sistema}

\section{Introducción}

Esta etapa identifica patrones comunes de comportamiento comunes que se repiten en el seno de los sistemas alimentarios. Al descubrir estos patrones, es posible entender cómo se comporta el sistema e identificar mejor y más rápido las palancas de cambio. Cuando vemos que e comportamiento del sistema se compone de patrones que se repiten a lo largo del tiempo y de las zonas geográficas, podemos identificar y anticiparnos mejor a los problemas y adaptarnos a ellos. En esta etapa, recurrimos a ocho patrones comunes, que se denominan arquetipos del sistema (Kim 1994).

\section{Breve descripción en las herramientas}

No hay herramientas estándar para desarrollar o identificar los arquetipos del sistema. El campo de las dinámicas del sistema reconoce varios comportamientos arquetípicos de los sistemas, de los que elegimos ocho que son pertinentes para los sistemas alimentarios. El reconocimiento de los arquetipos del sistema se construye en gran medida sobre los diagramas de bucle causal, elaborados en la etapa previa y que identifican a las víctimas del sistema. Para cada serie de bucles causales, esta etapa identifica los arquetipos que determinan patrones recurrentes en el sistema alimentario.

Puesto que cada arquetipo tiene su propia dinámica en la situación concreta, la siguiente etapa consiste en estudiar en más detalle la situación con la lupa que nos brinda el arquetipo. Esto generará datos para poder entender por qué ocurre la problemática actual y cómo se pueden modificar estos patrones del comportamiento del sistema. Puesto que buena parte de esto exige interpretaciones por parte de los investigadores, recomendamos celebrar un taller con grupos de interés y expertos para validar este análisis, que ayudará a identificar las palancas que pueden ayudar a conseguir un cambio transformador del sistema alimentario.

El campo de las dinámicas del sistema reconoce varios comportamientos arquetípicos de los sistemas. Aquí, ponemos cuatro ejemplos. Posthumus et al. (2018b) explica con más detalle ocho arquetipos de los sistemas alimentarios.

\section{Principios de calidad}

Los diagramas de bucle causal y los arquetipos del sistema no siempre distinguen las diferencias de cómo se ven afectados distintos grupos sociales. Por eso es importante incluir en los talleres grupos de interés o informantes clave que representen o conozcan suficientemente cómo afectan las distintas dinámicas a los diferentes grupos sociales. Para más información, véanse los capítulos sobre la implicación de los grupos de interés y sobre igualdad e inclusión.

\section{Éxito para los que tienen éxito}

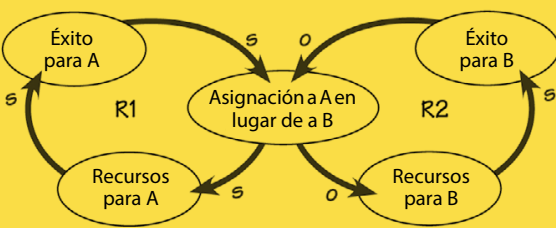

Se dota a un grupo de más recursos - Más probabilidades de éxito - La inversión atrae la inversión

\section{Ejemplo: Competencia entre empresas} Las start-ups del sector agricola que obtienen más subvenciones al principio tienen más probabilidades de éxito que otras empresas

\section{Limites del éxito}

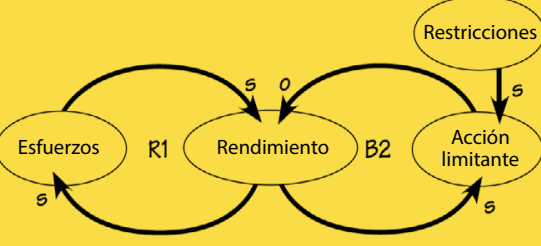

- El rendimiento aumenta - Posteriormente, el sistema se topa con un límite

- El rendimiento se reduce o erosiona

Ejemplo: Nutrición

El aumento de la diversidad en la nutrición genera efectos positivos hasta un determinado punto de satisfacción

\section{Soluciones que no funcionan}

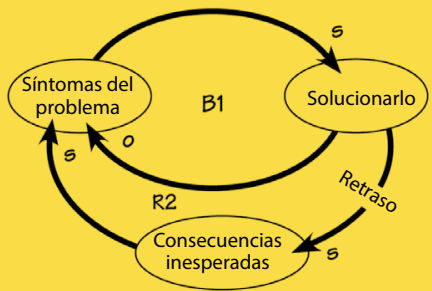

- Soluciones a corto plazo para problemas a largo plazo

- Las soluciones rápidas solo hacen más grande el problema solución

Ejemplo: fertilizante inorgánico

Supone una ventaja para la fertilidad del suelo a corto plazo, pero no supone ninguna inversión en gestión integrada del suelo con el fin de regenerarlo. Mayor dependencia de los insumos a largo plazo.

\section{Tragedia de los comunes}

- Un comportamiento beneficia al individuo - La comunidad no se beneficia La comunidad se deteriora

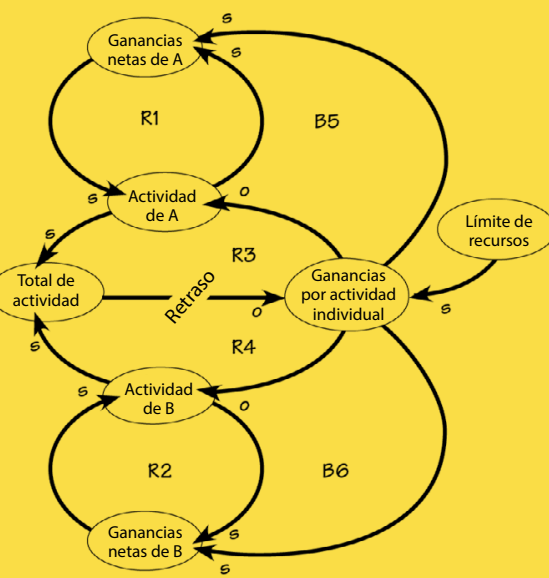

Ejemplo: Deforestación

Los agricultores utilizan servicios forestales. Si hay demasiada presión, la masa forestal se acabará deteriorando. 


\section{Herramienta 3: Identificación de las palancas de cambio}

\section{Introducción}

Una vez definidos los problemas sistémicos, se identifican palancas de cambio que puedan modificar un comportamiento del sistema que genere problemas. Una palanca se entiende como un lugar en el seno de un sistema donde el giro de un factor puede tener un efecto catalizador del cambio en todo el sistema alimentario. Estas palancas pueden incluir la mejora de los flujos de información, el fortalecimiento o debilitamiento de circuitos de retroalimentación, o la puesta en marcha de nuevos incentivos para influir sobre el comportamiento de los actores.

\section{Breve descripción en las herramientas}

En función de los mecanismos del bucle causal y los arquetipos del sistema, el análisis empieza por identificar qué debería cambiar para lograr un resultado (más) positivo. Cuando se elaboran las posibles soluciones, se analiza cómo influirian en el resto del sistema alimentario y se emplean para analizar si una meta se puede alcanzar y cómo. Para profundizar en el análisis, recomendamos recurrir al modelo bayesiano. Este modelo ayuda a predecir cómo afectarán los cambios que se perciben a otros aspectos del sistema alimentario.

Se recomienda clasificar las palancas en función de su relevancia y potencial para lograr los resultados del sistema alimentario que se deseen, preferiblemente, de la mano de los grupos de interés clave. Para ello, podemos utilizar las herramientas de exploración. Tenga también en cuenta qué grupos de interés o actores influyen sobre, o se ven inmediatamente afectados por, estas palancas. Estas perspectivas fundamentarán el tipo de colaboraciones o incentivos necesarios para activar las que se hayan identificado. El siguiente apartado, que versa sobre los actores del sistema, ofrece más información.

\section{Principios de calidad}

Tenga en cuenta que los efectos de las palancas pueden variar entre los diferentes grupos sociales. La elección de palancas puede ser distinta en función de los objetivos que se hayan formulado al empezar el análisis. Por ejemplo, las palancas que pueden hacer el sistema alimentario más inclusivo pueden ser distintas de aquellas que podrian incrementar la rentabilidad o los beneficios.

\begin{tabular}{|c|c|c|c|c|c|c|c|c|}
\hline \multicolumn{3}{|c|}{ (2) Herramientas de concienciación } & \multicolumn{3}{|c|}{ OO Herramientas de exploración } & \multicolumn{3}{|c|}{ Allitherramientas de análisis } \\
\hline $\begin{array}{l}\text { Ruptura del ciclo } \\
\text { En función de las } \\
\text { dinámicas de los } \\
\text { arquetipos, trate de } \\
\text { encontrar maneras de } \\
\text { alterar los ciclos para } \\
\text { lograr resultados (más) } \\
\text { positivos. ¿Qué deberia } \\
\text { cambiar en las diná- } \\
\text { micas para hacerlas } \\
\text { positivas o negativas? }\end{array}$ & $\begin{array}{l}\text { Tormenta de ideas } \\
\text { sobre innovación } \\
\text { Ejercicio de tormenta } \\
\text { de ideas sobre la } \\
\text { ruptura del ciclo. Los } \\
\text { participantes res- } \\
\text { ponden a la pregunta: } \\
\text { ¿cómo podemos hacer } \\
\text { realidad este cambio? } \\
\text { Ofrezca margen para } \\
\text { las soluciones que ya } \\
\text { hay y para ideas creati- } \\
\text { vas y novedosas. }\end{array}$ & $\begin{array}{l}\text { Rueda de futuros } \\
\text { Herramienta visual } \\
\text { para hacer una tormen- } \\
\text { ta de ideas sobre las } \\
\text { consecuencias direc- } \\
\text { tas de los cambios que } \\
\text { se propongan. }\end{array}$ & $\begin{array}{l}\text { Planificación para } \\
\text { el éxito } \\
\text { Mapeo de los efectos } \\
\text { de un circuito de } \\
\text { retroalimentación } \\
\text { cambiante sobre el } \\
\text { resto del sistema } \\
\text { alimentario. Tenga en } \\
\text { cuenta las diferencias } \\
\text { en la velocidad a la } \\
\text { que se pueda notar el } \\
\text { impacto y los limites } \\
\text { de crecimiento. Ofrece } \\
\text { aportes directos para } \\
\text { el diseño de politicas. }\end{array}$ & $\begin{array}{l}\text { Análisis SWOT } \\
\text { Esta herramienta de } \\
\text { evaluación genera una } \\
\text { lista de fortalezas, de- } \\
\text { bilidades, oportunida- } \\
\text { des y amenazas de un } \\
\text { proyecto, colaboración } \\
\text { o producto. Esto ayuda } \\
\text { a distinguir los factores } \\
\text { en los que se puede } \\
\text { influir de los que no. }\end{array}$ & $\begin{array}{l}\text { Validación de los } \\
\text { grupos de interés } \\
\text { Esta herramienta } \\
\text { es especialmente } \\
\text { importante cuando los } \\
\text { grupos de interés solo } \\
\text { se hayan implicado } \\
\text { parcialmente en } \\
\text { las herramientas } \\
\text { anteriores de esta } \\
\text { etapa. Pregunte a } \\
\text { los grupos de interés } \\
\text { qué les suena, qué les } \\
\text { sorprende y qué echan } \\
\text { en falta. Céntrese en su } \\
\text { análisis de las distintas } \\
\text { palancas de cambio del } \\
\text { sistema alimentario. } \\
\text { åf } \\
\text { 1-10 dias }\end{array}$ & $\begin{array}{l}\text { Modelo bayesiano } \\
\text { Cree un modelo del } \\
\text { efecto de dinámicas } \\
\text { cambiantes tal y como } \\
\text { las haya planificado, } \\
\text { es probable que se } \\
\text { materialicen utilizando } \\
\text { el modelo bayesiano. }\end{array}$ & $\begin{array}{l}\text { Mapa cognitivo } \\
\text { difuso } \\
\text { Técnica de modelado } \\
\text { que mapea los vínculos } \\
\text { causales y los efectos } \\
\text { esperados sobre la } \\
\text { base de entrevistas a } \\
\text { expertos. El modelo } \\
\text { calcula cómo afecta } \\
\text { a todo el sistema el } \\
\text { cambio en una variable. }\end{array}$ & $\begin{array}{l}\text { SUSFANS } \\
\text { Herramienta de } \\
\text { modelado que trata de } \\
\text { predecir cómo se com- } \\
\text { portarán en un futuro } \\
\text { los actores clave en } \\
\text { cuanto a la respuesta } \\
\text { de unos a otros, por } \\
\text { lo que hace posible } \\
\text { una evaluación de las } \\
\text { posibles intervencio- } \\
\text { nes en pro de una dieta } \\
\text { saludable en Europa. }\end{array}$ \\
\hline
\end{tabular}




\section{Del análisis a la acción}

Índice

- Decisión de los puntos de entrada de la intervención

- Implicación de los grupos de interés para identificar sendas de transformación

- El análisis del sistema alimentario, en acción 


\section{Decisión de los puntos de entrada de la intervención}

Una vez analizadas las tres dimensiones del sistema alimentario (actores del sistema, características de sistema y comportamiento del sistema), podemos elegir en qué punto del sistema queremos intervenir. A continuación, ofrecemos algunas preguntas e indicaciones para guiar esta etapa.

\section{Identificación de bolsas de resistencia al cambio}

¿Existen circuitos de retroalimentación o arquetipos persistentes que haya que cambiar? Es posible

distinguir medios para la transformación o para lograr un cambio progresivo:

Transformación del sistema: elimine o añada circuitos de retroalimentación a la estructura del sistema

Cambio progresivo: ralentización o aceleración de circuitos de retroalimentación específicos

- ¿Existen grupos de interés o instituciones con poder para evitar el cambio?

¿Cómo puede corregirse esa situación?

- En función de lo anterior, ¿cuáles son las posibles palancas de cambio?

- ¿Con qué grupos de interés importantes podría colaborar?

\section{Identificación de los problemas a corto, medio y}

largo plazo que hay que corregir

Piense en las acciones adecuadas que podría poner en marcha al tiempo que presta atención a las sinergias y equilibrios que las diferentes acciones pueden generar a lo largo del tiempo:

¿Qué posibilidades hay de reaccionar a problemas urgentes a corto plazo?

- ¿Qué desafíos a medio plazo pueden anticiparse?

¿Qué desafíos a largo plazo exigen una revisión de

diseño de las estructuras del sistema o un cambio en

nuestros modelos mentales?
Búsqueda de sinergias con los resultados y la propia cartera de actividades, intervenciones 0 políticas

¿Qué partes de la cartera deberían interrumpirse?

¿Qué partes de la cartera deberían cambiar o mejorar?

- ¿Qué partes de la cartera deberían multiplicarse?

- ¿Qué partes de la cartera deberían incorporarse?

\section{Tenga en cuenta los cerrojos en esta etapa}

Los cerrojos son aquellas situaciones que mantienen el sistema en su estado actual e impiden su transformación. El IPES (2016) ha señalado los siguientes cerrojos, que pueden crear resistencia a los cambios drásticos en los sistemas alimentarios de los países industrializados:

Dependencia de sendas. Los actuales sistemas alimentarios tienden a reforzarse.

Orientación a la exportación. Las políticas y acuerdos comerciales agrícolas priorizan la exportación de las materias primas agroalimentarias.

Expectativa de alimentos baratos. Procesadoras y distribuidores dependen del suministro barato y flexible de materias primas harmonizadas.

Pensamiento compartimentado. Las instituciones (política, investigación, empresa) se han compartimentado según el modelo de la agricultura industrializada.

Pensamiento a corto plazo. Las soluciones a corto plazo dominan debido a los ciclos electorales y los intereses comerciales.

Relatos de dar de comer al mundo. Centrarse en el total de alimentos que se producen es perjudicial para la igualdad social, los desequilibrios de poder o la disponibilidad de alimentos y el acceso a ellos.
Medición de éxito. El desempeño se mide sobre todo en función del rendimiento y la productividad de las cosechas, en vez de a través de indicadores como la resiliencia o los factores externos.

Concentración de poder. Un reducido número de multinacionales dominan el suministro de insumos, y los sectores del procesamiento y la distribución de alimentos. 


\section{Implicación de los grupos de interés para identificar sendas de transformación}

"Es realmente difícil utilizar las previsiones para prever cuál será la dirección del cambio. Al fin y al cabo, nadie sabe lo que pasará en el futuro. En los últimos diez años, hemos aprendido mucho más sobre las transiciones agroalimentarias y su funcionamiento. Sobre por qué hay innovaciones que se aplican a una escala más grande y otras nunca se adoptan mayoritariamente."

En esta herramienta, tratamos con detalle la importancia de implicar a diversos grupos de interés a lo largo de todo el proceso (véase el Principio de calidad B). La implicación de los grupos de interés también es importante en la etapa de identificación de sendas de transformación. De este modo, no solo se fundamentarán las decisiones sobre futuros inciertos, también se creará agencia.

Un método para implicar a los grupos de interés en esta etapa es la fórmula de los tres horizontes (Sharpe et al. 2016). Este método ofrece un marco para el diálogo estructurado y guiado con los grupos de interés. El diálogo dará lugar a un intenso debate y a que surjan desencuentros, y pondrá de manifiesto las brechas de conocimiento sobre el futuro. El principal objetivo es, no obstante, identificar posibles acciones que supongan dar pasos por la senda del cambio.

El marco trabaja con tres horizontes temporales.

- El Horizonte Uno (H1) representa la forma de hacer las cosas en este momento (las actividades ordinarias). El punto de partida del debate es el reconocimiento de que este horizonte está perdiendo brillo con motivos de las nuevas situaciones que aparecen (ej.: cambio climático, cambios sociales o tecnológicos) o los resultados deseados.
- El Horizonte Tres (H3) es la visión o el horizonte a largo plazo que pretendemos conseguir con el fin de gestionar las oportunidades y/o desafíos que presentan esas nuevas situaciones.

- Horizonte Dos (H2) es la etapa de transición turbulenta que hay entre el primer y el tercer horizonte. Es aquí donde se desafían o disrumpen las estructuras actuales, y donde surgen las innovaciones.

Con las perspectivas obtenidas a partir del análisis de sistema alimentario, $y$, en particular, las palancas de cambio del sistema que haya identificado, puede debatir los distintos horizontes para identificar posibles sendas de transformación.

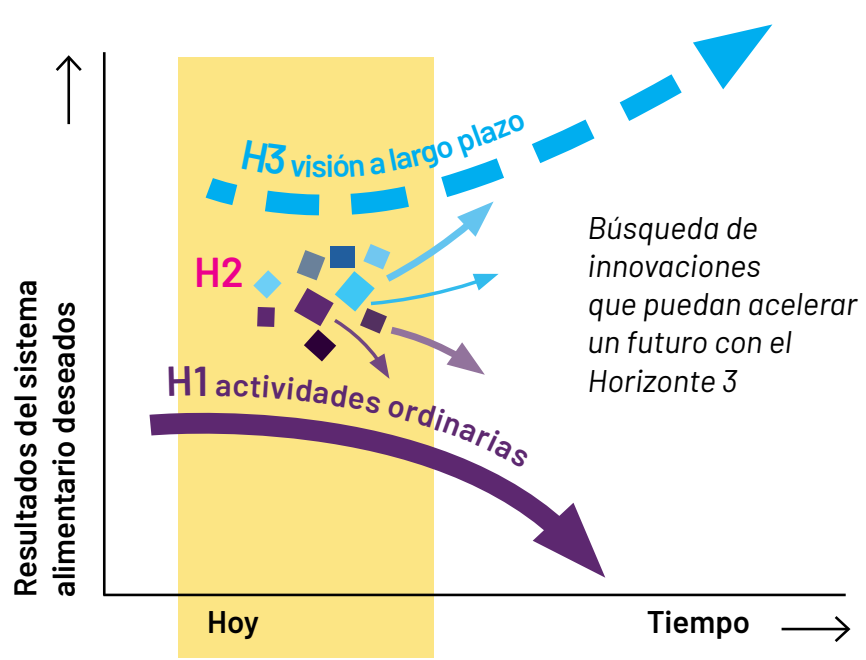

El diálogo de los Tres Horizontes puede facilitarse siguiendo varios pasos:

1. Analizar las preocupaciones actuales (H1): debatir cómo la manera de trabajar actual está quedando obsoleta debido a las nuevas situaciones.

2. Analizar las aspiraciones de cara al futuro (H3): analizar las aspiraciones y visiones, así como los aspectos del $\mathrm{H} 1$ que deben cambiar.

3. Explorar las prácticas inspiradoras que se dan en la actualidad: buscar ejemplos concretos en los que ya sean marginalmente visibles las nuevas formas de hacer negocio (bolsas de futuro en el presente).

4. Identificar innovaciones (H2): buscar innovaciones que den respuesta a los fallos del $\mathrm{H} 1$ y las posibilidades del $\mathrm{H} 3$.

5. Determinar características esenciales que deban mantenerse: identificar elementos del antiguo sistema (H1) que deban mantenerse en el futuro (H3).

Una manera de analizar esto más en profundidad es hacerlo a través del Marco de Perspectiva Multinive (PMN)(Geels y Schot, 2007). Explica cómo se manifiestan las transiciones a través de la interacción entre los tres niveles del análisis en los sistemas sociales: el paisaje, los regímenes y los nichos. 


\section{El análisis del sistema alimentario, en acción}

\section{Elección de las mejores opciones para actuar}

El análisis del sistema alimentario debería dar como resultado una mejor comprensión de los motores y grupos de interés que influyen en todo el sistema alimentario. Una vez identificadas las palancas para el cambio sistémico, habrá que elegir aquellas que puedan traducirse en recomendaciones prácticas de cara a la estrategia y las intervenciones.

Esta última etapa del proceso explora el margen que existe para posibles intervenciones dirigidas a estas palancas. Este espacio queda determinado por las capacidades de la parte interviniente (ej.: legislador u ONG), los efectos anticipados de las intervenciones sobre distintos grupos de interés, y el margen de intervención de otros actores.

Si tomamos como referencia el enfoque de diseño centrado en las personas, podemos hacernos estas tres preguntas para centrar las recomendaciones: ¿Qué quieren las personas? ¿Qué es económicamente viable? ¿Qué es técnica y organizativamente factible?

\section{Posibles criterios para elegir:}

- Relevancia, nivel de impacto y sostenibilidad del cambio sistémico para distintos grupos sociales.

- Las sinergias y equilibrios de las distintas opciones para distintos grupos sociales.

- Fortalezas, conocimiento experto y recursos de la parte interviniente, pero también, mandato y legitimidad para iniciar el cambio.

- Eficiencia económica de las intervenciones.

- Valor añadido de la estrategia en relación con las iniciativas, intervenciones o políticas que influyen sobre el sistema alimentario que se aplican actualmente

- Potencial de creación de sinergias con otras iniciativas o socios existentes.

- Equilibrio entre fuerzas (grupos de interés) a favor y en contra de su estrategia, y su capacidad para influir sobre ellas.

Medida en la que los supuestos y riesgos subyacentes a la estrategia son razonables, aceptables y manejables.

\section{Existen varias herramientas que ayudan a priorizar y elegir}

Priorización y clasificación Esta herramienta le ayudará a elegir aquellas ideas u opciones más prometedoras en los casos en los que haya surgido un gran número de ellas.

Debate las opciones en grupo, y se votan las mejores ideas.

Delphi Cuando se afronten cuestiones complejas, invite a un panel de expertos. Recopile y resuma sus respuestas y compártalas. Formule al panel la misma pregunta nuevamente y compruebe si se crea un consenso.
Comparación de propuestas Esta herramienta es una simple lista para valorar propuestas desde distintos puntos de vista. Capta las propuestas alternativas elaboradas por el grupo, y analiza los equilibrios y sinergias correspondientes. Un análisis multicriterio puede ser más elaborado (tenga en cuenta que existen muchos métodos) mediante la comparación de distintas propuestas con una serie de criterios diversos.

¿A quién se dirige la política? ¿Qué tipos de resultados socioeconómicos del sistema alimentario tratamos de cambiar y para quién? Explore los resultados de género deseados para las intervenciones (ámbito, beneficio, empoderamiento, transformación).

Análisis del campo de fuerzas Esta herramienta evalúa las fortalezas de varias fuerzas favorables y contrarias a un cambio deseado que influyen sobre él. Puede fundamentar decisiones sobre qué colaboraciones o coaliciones crear para lograr el cambio.

Elaboración de una Teoría del Cambio Defina cómo va a ser la estrategia, qué actividades y recursos son necesarios, con quién colaborar y cómo se espera que esta estrategia conduzca al cambio. Incluya en la Teoría del Cambio el impacto sobre distintos grupos sociales y los supuestos y riesgos subyacentes.

Consulte a los grupos de interés a lo largo de todo el proceso para validar la lógica de su cambio. Hay varias formas de obtener retroalimentación, como jurados populares, testeo de campo y encuestas de retroalimentación. Hay que prestar especial atención para garantizar que se consulta a personas con diversos perfiles y que la modalidad de consulta facilita que se puedan escuchar todas las voces. 


\section{Panorámica general de las fuentes}

\section{Artículos e informes}

Brouwer, H., and J. Woodhill; with M. Hemmati, K. Verhoosel, S. van Vugt. 2016. The MSP Guide, How to design and facilitate multi-stakeholder partnerships, Wageningen: Wageningen University an Research, CDI, and Rugby, Reino Unido: Practical Action Publishing. www.mspguide. org

Danielsen, K., F.F. Wong, D. McLachlin, S. Sarapura. 2018. Typologies of Chang: Gender Integration in Agriculture and Food Security Research. Ámsterdam: KIT Royal Tropical Institute

Dekeyser, K., F. Rampa, C. D'Alessandro, P. Bizotto Molina. 2020. The food systems approach in practice: our guide for sustainable transformation. Discussion paper 278. European Centre for Development Policy Management, Maastricht

Geels, F., Schot, J., 2007. Typology of transition pathways in socio-technical systems. Research Policy.

IPES. 2016. From uniformity to diversity: paradigm shift from industrial agriculture to diversified agroecological systems.

International Panel of Experts on

Sustainable Food systems. www.ipes-food. org

Kim, D.H. 1994. Systems archetypes II: using systems archetypes to take effective action. Pegasus Communications Inc
KIT e IIRR. 2010. Value chain finance: Beyond microfinance for rural

entrepreneurs. Royal Tropical Institute, Amsterdam; e International Institute of Rural Reconstruction, Nairobi.

Meadows, D. 1999. Leverage Points: Places to Intervene in a System. The Sustainability Institute, 2-19.

Medium. 2020. Disruptive design. https:// medium.com/disruptive-design/archive. Consultado el 30/09/2020

Newton, J. 2020. Gender, nutrition and food system approaches. En: C.E. Sachs, L. Jensen, P. Castellanos, K. Sexsmith (Eds.) Routledge Handbook of Gender and Agriculture. Routledge, Londres

Omidyar Group (2017) Systems Practice Workbook. https://docs.kumu.io/content/ Workbook-012617.pdf

Pegasus communication Inc. 2020 The Systems Thinker. https://

thesystemsthinker.com. Leverage Networks Inc. Consultado el 30/09/2020

Posthumus, H., B. de Steenhuijsen Piters, J. Dengerink, S. Vellema. 2018a. Food systems: from concept to practice and vice versa. Wageningen University \& Research, KIT Royal Tropical Institute

Posthumus, H., B. de Steenhuijsen Piters, J. Dengerink, S. Vellema. 2018b. Archetypes: common systemic behaviours in food systems. Wageningen University \& Research, KIT Royal Tropical Institute
Sharpe, B., A. Hodgson, G. Leicester, A. Lyon, I. Fazey. 2016. Three horizons: a pathways practice for transformation. Ecology and Society 21(2): 47

Termeer, C.J.A.M, S. Drimie, J. Ingram, L. Pereira, M.J. Whittingham. 2018. A diagnostic framework for food system governance arrangements: The case of South Africa. Wageningen: NJAS Wageningen Journal of Life Sciences. Vol 84, p 85-93.

The Rockefeller Foundation. 2019 Food System Vision Prize Envisioning: Regenerative and Nourishing Food Futures for 2050 - Toolkit.

Van Berkum, S, J. Dengerink, R. Ruben. 2018. The food system approach:

sustainable solutions for a sufficient supply of healthy food. Wageningen Economic Research, Memorandum 2018-064
Bases de datos y herramientas disponibles online

Demographic and Health Surveys (DHS). https://dhsprogram.com/methodology/ Survey-Types/DHS.cfm

Good Life Index. https://goodlife.leeds. ac.uk/

Modelo donut de Kate Raworth. www. kateraworth.com/doughnut/

\section{Mural. www.mural.co/templates}

Tablero de los Sistemas Alimentarios. https://foodsystemsdashboard.org/

Banco Mundial: Datos por paises. https:// data.worldbank.org/country

Banco Mundial: Doing business. www. doingbusiness.org/en/doingbusiness

\section{Fuentes de herramientas}

\section{Diagrama de afinidad}

www.burgehugheswalsh.co.uk/Uploaded/1/ Documents/Affinity-Diagram-Tool-Draft-v2. pdf

Modelos basados en agentes www.pnas.org/content/99/suppl_3/7280

\section{Comportamiento a lo largo del tiempo}

https://thesystemsthinker.com/a-paletteof-systems-thinking-tools/

\section{Ruptura del ciclo}

https://thesystemsthinker.com/wpcontent/uploads/2016/03/SystemsArchetypes-I-TRSA01_pk pdf

\section{Diagrama de circuito causal} https://thesystemsthinker.com/causal-

loop-construction-the-basics/

\section{Comparación de propuestas}

www.mspguide.org/tool/comparingproposals

Delphi

www.mspguide.org/tool/delphi

\section{Análisis donut}

ww.c40knowledgehub.org/s/article/ Creating-City-Portraits-A-methodologicalguide-from-the-Thriving-Cities-

nitiative?language=en_US

Las cinco A: implicaciones politicas https://ecdpm.org/publications/doingregional-development-differently/

Un paseo por la seguridad alimentaria www.mspguide.org/tool/food-security- 
Marco de relaciones de gobernanza del sistema alimentario www.sciencedirect. com/science/rticle/pii/S157352141730012X

\section{Análisis del campo de fuerzas}

www.odi.org/sites/odi.org.uk/files/odi-

assets/events-documents/2808.pdf \&

www.mspguide.org/tool/force-field-

analysis

\section{Formas de poder}

www.mspguide.org/tool/forms-power

\section{Futuros escenarios}

www3.weforum.org/docs/IP/2016/NVA/

WEF_FSA_FutureofGlobalFoodSystems.pd

\& http://www.mspguide.org/tool/scenarioplanning

\section{Rueda de futuros}

www.mindtools.com/pages/article/futureswheel.htm

\section{Mapa cognitivo difuso}

www.mentalmodeler.org/

\section{Indicadores de gobernanza}

https://info.worldbank.org/governance/ wgi/Home/Reports

Diagrama de función gráfica

https://thesystemsthinker.com/graphicalfunctions-seeing-the-full-story $/$

Inmersión a través del acompañamiento www.designkit.org/methods/immersion

Tormenta de ideas sobre innovación content/uploads/2 content/uploads/2016/03/SystemsArchetypes-I-TRSA01_pk.pdf
Herramienta integrada de evaluación de la biodiversidad

www.ibat-alliance.org/

\section{Mapa interactivo}

www.researchgate.net/

publication/333895085_Mapping_Food_

Systems_A_Participatory_Research_Tool

Tested_in_Kenya_and_Bolivia

\section{Matriz de importancia e influencia}

www.mspguide.org/tool/stakeholder-analysis-importanceinfluence-matrix

\section{Entrevistas}

www.mspguide.org/tool/semi-structured-interviews

\section{Mapeo del trayecto}

https://qualaroo.com/customer-jour-

ney-map-template/

\section{Mapa conceptual}

www.groupmap.com/map-templates/ group-mind-mapping/

\section{Coste minimo de una dieta saludable}

www.motherchildnutrition.org/malnutrition/pdf/mcn-stc-minimum-cost-of-ahealthy-diet.pdf

Evaluación de necesidades www.fao.org/3/w5830e07.htm

\section{Análisis de la brecha nutriciona} www.wfp.org/publications/2017-fill-nutrient-gap

\section{Una cadena de valor que tiene en cuenta la} nutrición

www.ifad.org/en/web/knowledge/publication/asset/40805038
Gráfico radial de los objetivos

www.fusioncharts.com/resources/

chart-primers/radar-chart

Mapeo del uso participativo de la tierr www.ifad.org/en/web/knowledge/publication/asset/39399099

\section{Planificación para el éxito}

https://thesystemsthinker.com/wp-content/uploads/2016/03/Systems-Archetypes-III-TRSA03_pk.pdf

\section{Análisis de las políticas}

www.ncchpp.ca/60/Analyzing_Public_Policies.ccnpps

\section{¿A quién se dirige la política?}

www.ifpri.org/blog/reach-benefit-or-empower-clarifying-gender-strategies-development-projects

\section{Evaluación de la economía política}

http://documents.worldbank.org/curated/ en/327051468337182275/pdf/769210W-

POPolit00Box374391BOOPUBLICO pdf

\section{Análisis de poder}

www.powercube.net/wp-content/ uploads/2009/11/quick_guide_to_power_ analysis_external_final.pdf

\section{Mapeo de poder}

www.mspguide.org/tool/netmapping

\section{Clasificación de poder}

www.mspguide.org/tool/power-ranking

Priorización y clasificación

www.mspguide.org/tool/prioritizing-and-ranking
Definición de problemas

www.mspguide.org/tool/problem-definition-worksheet

\section{Árbol de problemas}

www.mspguide.org/tool/problem-tre

\section{Gráfico 00}

https://thesystemsthinker.com/a-paletteof-systems-thinking-tools/

\section{Evaluación rápida del sector}

www.wur.nl/en/Research-Results/

Research-Institutes/centre-for-development-innovation/Our-Value-Proposition

Guiding-Sector-Transformation/Theeffects-of-COVID-19-on-food-systems-rapid-assessments.htm

\section{Ambito, beneficio o empoderamiento} www.ifpri.org/blog/reach-benefit-or-empower-clarifying-gender-strategies-development-projects

Cuadro enriquecido

www.mspguide.org/tool/rich-picture

Sensemaker

https://sensemaker.cognitive-edge.com/

\section{Análisis de la red social}

https://towardsdatascience.com/how-toget-started-with-social-network-analysis$6 \mathrm{~d} 527685 \mathrm{~d} 374$

Identificación de los grupos de interés www.mspguide.org/tool/stakeholder-identification

\section{Caracteristicas y roles de los grupos de}

\section{interés}

www.mspguide.org/tool/stakeholder-characteristics-and-roles-matrix
El poder de los grupos de interés en los sistemas alimentarios

\section{Análisis SWOT}

www.mspguide.org/tool/swot-analysis

\section{Sinergias y equilibrios}

Www.frontiersin org/articles/10.3389/fenvs.2018.00112/full \&

https://drift.eur.nl/publications food-and-agriculture-systems-foresight-study/

\section{Teoria del Cambio}

www.mspguide.org/tool/make-visualtheory-change

\section{Un paseo transversal}

https://catcomm.org/transect-walk/

\section{Clasificación de la riqueza}

www.padev.nl/other_output/PADev_guidebook_2013.pdf 


\section{Cierre}

Autores:

Helena Posthumus, Jonne Bosselaar y Herman Brouwer

Contribuciones: Bart de Steenhuijsen Piters, Ferko Bodnár, Julie Newton, Mona Dhamankar, Just Dengerink,

Simone van Vugt, Daphne Visser, Nina de Roo

\section{Cómo citar este documento:}

Posthumus, H., J.M. Bosselaar, H. Brouwer. 2021.

The food system decision support tool - a toolbox for food system analysis. Wageningen University \& Research y KIT Royal Tropical Institute.

(ㄷ) 2021 Wageningen Research y KIT Royal Tropical Institute

\section{Agradecimientos}

Los siguientes revisores externos han aportado observaciones útiles a una versión anterior: Frank Mechielsen (HIVOS), Koen Dekeyser (ECDPM), Michael Salomons (CFGB), Nicole Metz (F\&BKP), Rojan Bolling (F\&BKP), Rose Makenzi (EKN Kenia), Yvonne Mtumbi (AgriProFocus Zambia). También queremos dar las gracias a la Comunidad de Práctica sobre Sistemas Alimentarios moderada por Food \& Business Knowledge Platform (F\&BKP) por sus aportaciones.

Esta herramienta ha sido financiada por la Food \& Business Knowledge Platform (F\&BKP), que ha pasado a ser el Consorcio para la Alimentación de los Países Bajos,

www.nlfoodpartnership.com

Diseño: Roger Reuver (https://rco.design/
KIT Royal Tropical Institute

Mauritskade 64, 1092 AD Ámsterdam, Paises Bajos. $\mathbf{T}+31(0) 205688711$

Ecommunication@kit.nl

www.kit.nl.

\section{Wageningen Centre for Development Innovation}

parte de Stichting Wageningen Research

P.O. Box 88, 6700 AB Wageningen, Países Bajos $\mathbf{T}+31(0) 317486800$

Einfo.cdi@wur.nl

www.wureu/cdi

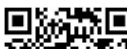

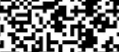 \\ rists}

Este informe puede descargarse gratuitamente en https://doi.org/10.18174/548819 o www.wur.eu/cdi (en Publicaciones).

El Wageningen Centre for Development Innovation utiliza una licencia Creative Commons Attribution 4.0 (Países Bajos) para sus informes.

\section{(cc) BY-NC}

